\title{
Introduction of hydroxyl- or keto- functionalities into the side chain of azetidin-2-ones via allylic bromide rearrangement, followed by supported reagent substitution
}

\author{
Fides Benfatti, Giuliana Cardillo,* Serena Fabbroni, Luca Gentilucci, Rossana \\ Perciaccante, and Alessandra Tolomelli \\ Dipartimento di Chimica "G. Ciamician" Università di Bologna, Via Selmi 2, \\ 40126 Bologna Italy \\ E-mail: giuliana.cardillo@unibo.it
}

\begin{abstract}
Dedicated to our friend Prof. Eusebio Juaristi on the occasion of his $55^{\text {th }}$ birthday (received 17 Mar 05; accepted 25 Apr 05; published on the web 27 Apr 05)
\end{abstract}

\begin{abstract}
This paper reports the allylic bromide rearrangement of 3-bromo-3-alkenyl-azetidin-2-ones, induced by $m$-chloroperbenzoic acid, $N$-bromosuccinimide or benzoylperoxide as radical initiators. The substitution of bromide by resin supported acids, followed by hydrolysis of the ester moiety, allowed an hydroxyl- or keto- function to be introduced in the C3 side chain of the azetidinone, thus giving access to a new class of potential cholesterol absorption inhibitors.
\end{abstract}

Keywords: Azetidinones, allylic bromide, rearrangement, supported reagents

\section{Introduction}

The nucleophilic substitution of allylic halides continues to draw great attention as it leads to a variety of derivatives, which can be converted into a wide spectrum of synthetically important molecules. ${ }^{1}$ Among the most interesting aspects of allylic compounds, the stereo- and regiochemistry of their reactions have received considerable attention.

In recent reports from our laboratory, the use of 3-bromo-3-alkenyl-azetidin-2-one as a particular allylic system has been studied. ${ }^{2}$ In fact, the bimolecular nucleophilic displacement with allylic rearrangement $\left(\mathrm{S}_{\mathrm{N}} 2\right.$ ' reaction) easily occurred on this kind of substrates either with azide anion $^{3}$ or under palladium catalyzed benzylamine attack. ${ }^{4}$ Both reactions afforded complete regio- and stereoselective substitution at $\mathrm{C} 2$ ' side chain with $\mathrm{C}-\mathrm{N}$ bond formation. The stereoselective anti $\mathrm{S}_{\mathrm{N}} 2$ ' attack of $\mathrm{NaN}_{3}$ to 3-alkenyl-3-bromo-azetidin-2-ones, followed by the stereoselective [3,3]-sigmatropic rearrangement of allylic azides, gave diastereomeric azides, useful precursors of 3(2'-amino)- $\beta$-lactams (Figure 1, pathway A). 


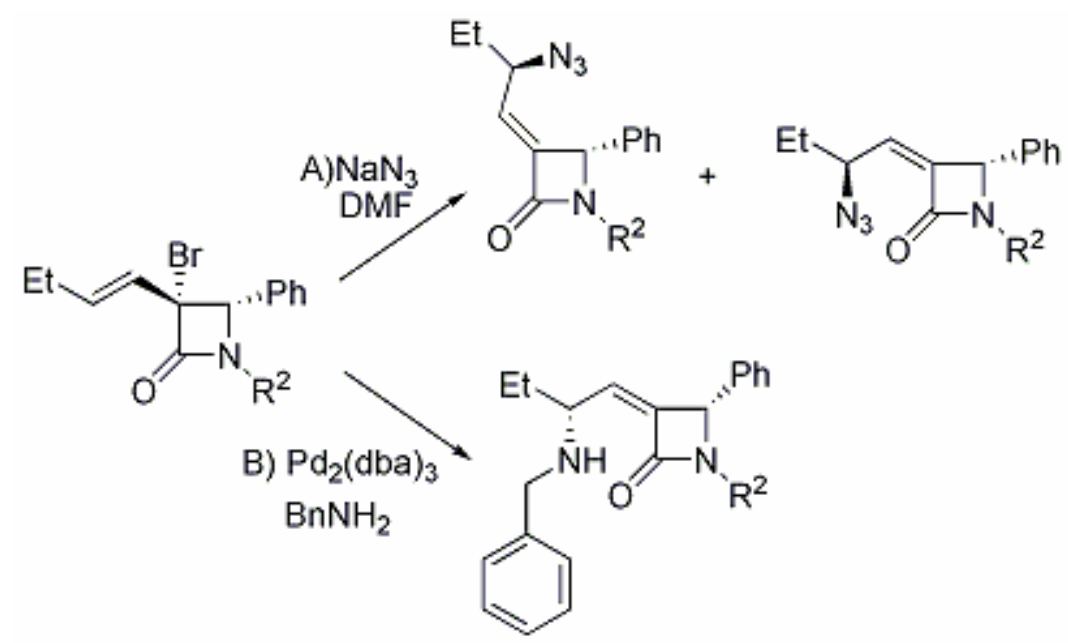

Figure 1. Reactions performed on 3-bromo-azetidin-2-ones.

On the other hand, palladium-catalyzed allylic substitution, provides one of the most efficient methods for the stereoselective construction of carbon-carbon or carbon-heteroatom bond. Therefore the palladium-catalyzed benzylamine attack to 3-alkenyl-3-bromo-azetidin-2ones allowed the amino function in the $\mathrm{C} 3$ side chain of the non conventional $\beta$-lactams to be introduced in a highly regio- and stereoselective fashion (Figure 1, pathway B).

The search for lead structures for the discovery of new protease inhibitors is a field of growing interest. Beside the high antibacterial activity showed by various natural $\beta$-lactams, new applications of azetidin-2-ones in the field of enzyme inhibition of proteases have been recently reported. ${ }^{5}$ The $\beta$-lactam ring represents the most important pharmacophore group for the inhibition activity, nevertheless the activity can be enhanced by proper functionalization at the side chain in $\mathrm{C} 3$ and $\mathrm{C} 4$ position of the ring.

\section{Results and Discussion}

During our investigation on the reactivity of $\alpha$-alkenyl- $\alpha$-bromo- $\beta$-lactams 1a-e, we observed an unusual behavior of these substrates. When they were treated with $m$-chloroperoxybenzoic acid (MCPBA) in 2M solution in $\mathrm{CH}_{2} \mathrm{Cl}_{2}$, the exclusive formation of the epoxides was observed. On the other hand a 40:60 mixture of epoxide and bromine rearrangement products, in $\mathrm{E}$ and $\mathrm{Z}$ configuration of the double bond, was observed when the reaction was stirred for three hours at r.t. under diluted conditions ( $1 \mathrm{mmol}$ of $1 \mathrm{a}$ with 1.5 equivalents of MCPBA in $5 \mathrm{~mL}$ of $\mathrm{CH}_{2} \mathrm{Cl}_{2}$ ).

The dependence of the reaction upon the dilution, suggested a radical mechanism. In agreement with recent reports ${ }^{6}$ that propose unsymmetrical biradical transition state in the epoxidation of the double bond performed with peracids, the possibility that MCPBA could act as radical initiator could explain the outcome of the reaction. 
Starting from the hypothesis of a radical mechanism, ${ }^{7}$ we treated compound $1 \mathbf{1 a}$ in refluxing 1,2-dichloroethane with benzoylperoxide (method A) or in $\mathrm{CH}_{2} \mathrm{Cl}_{2}$ with $\mathrm{N}$-bromosuccinimide at r.t. (method B), reagents that are well known radical initiators (Scheme 1). Under these conditions the same mixture of E- and Z-bromo derivateives was obtained in quantitative yield.
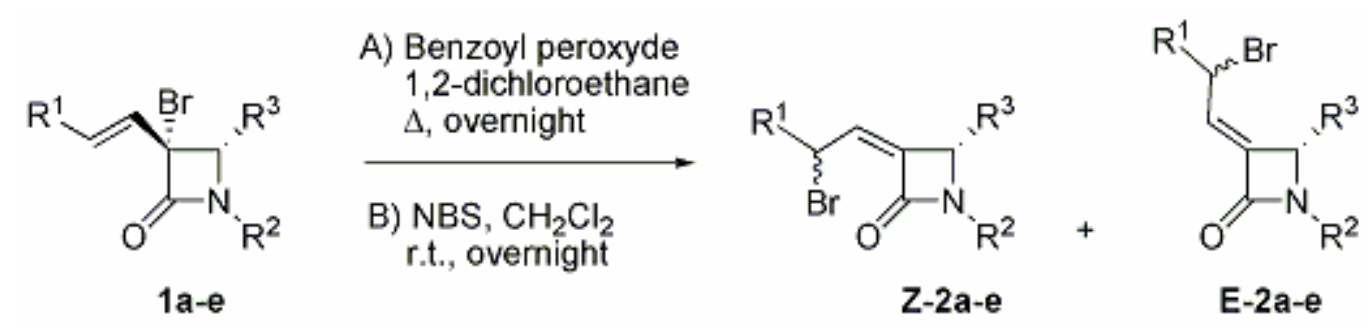

Scheme 1. Radical rearrangement of 1a-e with benzoylperoxide or $N$-bromosuccinimide.

The extraction of the organic phase afforded $80 \%$ of a $1: 1$ easily separable mixture of $Z$ and $\mathrm{E}$ diastereomers of 2'-bromolactam 2a. Each Z or E compound was obtained with a low control of the selectivity in the formation of the newly introduced stereogenic C2' centre (70:30). The stereochemistry on the newly introduced stereocentre was not investigated. The $\mathrm{Z}$ and $\mathrm{E}$ configuration was attributed on the basis of vinyl $\mathrm{H}_{1}$ chemical shift of the ${ }^{1} \mathrm{H}$ NMR spectrum, as reported in the literature. ${ }^{8}$ Then, we applied the same reaction to substrates $\mathbf{1 b}$-e and the results are reported in Table 1. Racemic mixtures of Z-2a-e and E-2a-e were separated by flash chromatography on silica gel.

Table 1. Rearrangement of 1 to $\mathrm{Z} / \mathbf{E}-2$

\begin{tabular}{cccccc}
\hline Entry $^{(\mathrm{a})}$ & & $\mathrm{R}^{1}$ & $\mathrm{R}^{2}$ & $\mathrm{R}^{3}$ & $\begin{array}{c}\text { Yield } \mathrm{Z}+\mathrm{E}^{\mathrm{a}} \\
(\%)\end{array}$ \\
\hline 1 & $\mathbf{1 a}$ & $\mathrm{H}$ & $\mathrm{CH}_{2} \mathrm{Ph}$ & $\mathrm{Ph}$ & 95 \\
2 & $\mathbf{1 b}$ & $\mathrm{Et}$ & $\mathrm{CH}_{2} \mathrm{Ph}$ & $\mathrm{Ph}$ & 97 \\
3 & $\mathbf{1 c}$ & $\mathrm{Et}$ & $\left(\mathrm{CH}_{2}\right)_{2} \mathrm{CO}_{2} \mathrm{Et}$ & $\mathrm{Ph}$ & 92 \\
4 & $\mathbf{1 d}$ & $\mathrm{Et}$ & $\mathrm{CH}_{2} \mathrm{Ph}$ & $\mathrm{p}-\mathrm{NO}_{2} \mathrm{Ph}$ & 78 \\
5 & $\mathbf{1 e}$ & $\mathrm{Et}$ & $(\mathrm{S})-\mathrm{phenylethyl}$ & $\mathrm{Ph}$ & 80 \\
\hline
\end{tabular}

${ }^{a}$ Equimolar amount of $\mathrm{Z}$ and $\mathrm{E}$ diastereomers were obtained with $70 / 30$ d.r. The diastereomeric mixtures were separated by flash chromatography on silica gel.

Aiming to introduce functionalities into the $\mathrm{C} 3$ side chain, we carried out a series of simple substitution reactions on Z-2 and E-2 derivatives. Many examples of $\beta$-lactam based cholesterol 
absorption inhibitors containing hydroxyl- and keto- functions in the $\mathrm{C} 3$ side chain have been recently reported in the literature. ${ }^{9}$ Therefore, in connection with our scientific interests, we developed an efficient and very mild process to convert allyl bromides to the corresponding alcohols. Each E-bromo derivatives E-2a-c was treated with benzoic acid supported on macroreticular styrene-divinylbenzene resin with quaternary ammonium functionality, for $2 \mathrm{~h}$ in benzene at reflux. ${ }^{10}$ Under the same reaction conditions $\mathbf{E - 2 b}$ and $\mathbf{E - 2 d}$ were treated with supported phenylacetic acid, with the aim to perform the enzymatic resolution of the racemic phenylacetates by treatment with penicillin $\mathrm{G}$ acylase. The bromides were easily substituted and the corresponding benzoates E-3a-c or phenylacetates E-4b-d were obtained in good yield with complete stereo- and regiocontrol (Scheme 2). It is well known that the supported reagents are very useful since they show high nucleophilicity, the reaction occurs under very mild conditions and in a variety of organic solvents, the usual work-up is avoided and it is reduced to the simple filtration of the resin and evaporation of organic solvents. ${ }^{11}$
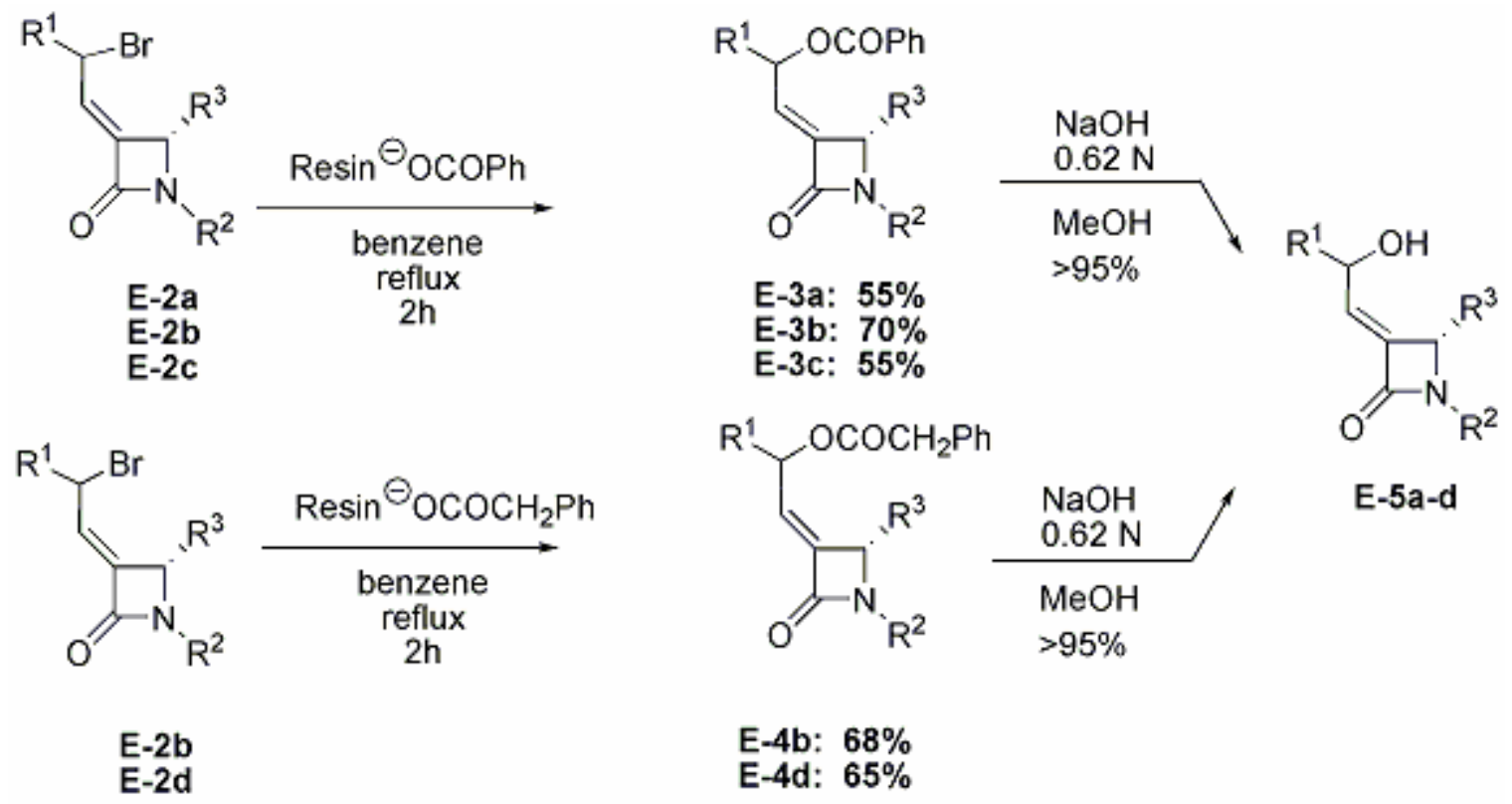

E-4b: $68 \%$

E-4d: $65 \%$

Scheme 2. Reaction of E-2 with resin supported benzoic acid and phenyl acetic acid followed by hydrolysis to E-5.

In a similar way, Z-2a-b afforded after treatment with benzoic acid supported on resin, the corresponding benzoates $\mathbf{Z}-\mathbf{3 a}$ and $\mathbf{Z}-\mathbf{3 b}$ in $87 \%$ and $70 \%$ yields respectively. By treating Z-2b and Z-2d with phenylacetate resin $\mathbf{Z - 4 b}$ and $\mathbf{Z - 4 d}$ were isolated in $72 \%$ and $55 \%$ yield respectively. In order to avoid the opening of the $\beta$-lactam ring the hydrolysis of the esters was carried out under mild conditions with $\mathrm{NaOH} 0.6 \mathrm{~N}$ in methanol. The hydrolysis smoothly occurred in $48 \mathrm{~h}$, affording the $\mathrm{E}$ or $\mathrm{Z}$ allylic alcohols 5a-d in quantitative yield. 
On pursuing the investigation on the reactivity of our substrates with supported reagents, we performed the oxidation of the alcohols 5 with chromic acid supported on anion exchange resin. ${ }^{12}$ Among the variety of procedures for the oxidation of alcohols, this method is remarkably efficient in the clean conversion of primary and secondary alcohols into aldehydes and ketones respectively. The reaction was tested on the primary alcohol Z- and E-5a and on the secondary E-5b. By refluxing Z-5a and E-5a in benzene, in the presence of 3 equiv. of chromic acid supported on anion exchange resin (loading $2.1 \mathrm{mmol} / \mathrm{g}$ ), the formation of the aldehyde Z-6a and E-6a in 53\% yield and 55\% yield respectively, was observed in $4 \mathrm{~h}$. Under the same reaction conditions, E-5b was quantitatively converted into ketone E-7b (Scheme 3).

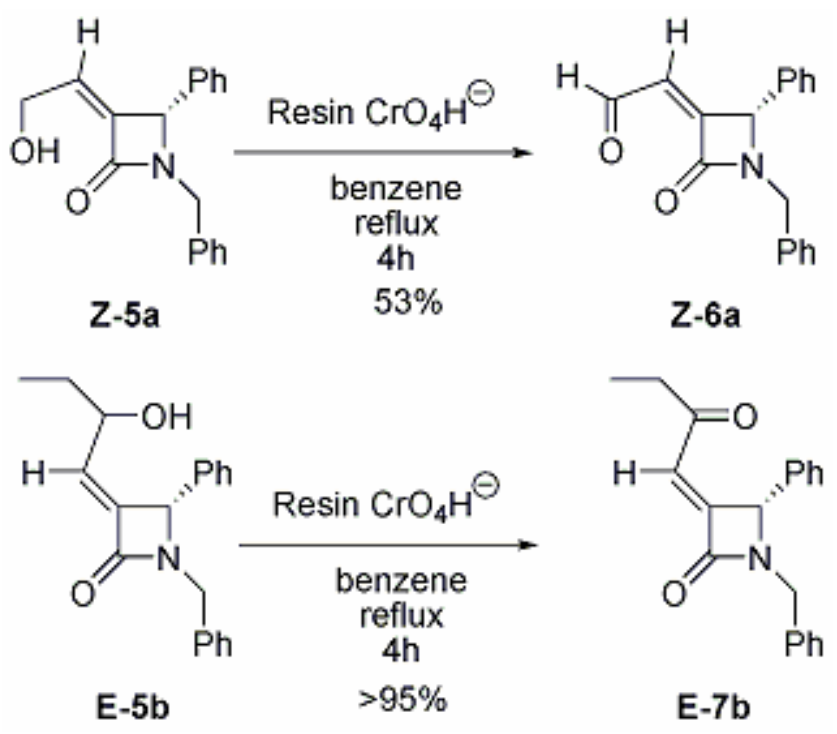

Scheme 3. Oxidation of hydroxy-derivatives Z-5a and E-6a to carbonyl derivatives.

In conclusion, this paper reports the allylic bromide rearrangement of 3-bromo-3-alkenylazetidin-2-ones, induced by MCPBA, NBS or benzoylperoxide as radical initiators. The substitution of bromide by resin supported acids followed by hydrolysis of the ester moiety, allows to introduce an hydroxyl- or keto- function in the $\mathrm{C} 3$ side chain of the azetidinone, thus giving access to a new class of potential cholesterol absorption inhibitors.

\section{Experimental Section}

General Procedures. Unless stated otherwise, solvents and chemicals were obtained from commercial sources and used without further purification. Flash chromatography was performed on silica gel (230-400 mesh). NMR Spectra were recorded with $300 \mathrm{MHz}$ spectrometers. Chemical shifts were reported as $\delta$ values (ppm) relative to the solvent peak of $\mathrm{CDCl}_{3}$ set at $\delta=$ $7.27\left({ }^{1} \mathrm{H}\right.$ NMR) or $\delta=77.0\left({ }^{13} \mathrm{C} \mathrm{NMR}\right)$. Infrared spectra were recorded with an FT-IR 
spectrometer. Melting points are uncorrected. MS analyses were performed on a liquid chromatograph coupled with an electrospray ionization-mass spectrometer (LC-ESI-MS), using $\mathrm{H}_{2} \mathrm{O} / \mathrm{CH}_{3} \mathrm{CN}$ as solvent at $25^{\circ} \mathrm{C}$ (positive scan $100-500 \mathrm{~m} / \mathrm{z}$, fragmentor $70 \mathrm{~V}$ ). Chiral HPLC analysis were performed on Chiralcel-OD Daicel column, Cellulose tris(3,5-dimethylphenyl)carbamate phase coated on $10 \mu \mathrm{m}$ silica gel, $n$-hexane/2-propanol 90:10 solvent mixture, flow $0.5 \mathrm{~mL} / \mathrm{min}$.

\section{General procedure for the rearrangement of 1a-e to Z/E-2a-e}

Method A: To a stirred solution of 1 (1 mmol) in 1,2-dichloroethane $(5 \mathrm{~mL})$, benzoylperoxide (1.1 mmol, 1.1. equiv., $266 \mathrm{mg}$ ) was added in one portion. The reaction was refluxed overnight and then quenched with water $(5 \mathrm{~mL})$. After dilution with 1,2-dichloroethane $(10 \mathrm{~mL})$, the organic layer was washed twice with water and dried over $\mathrm{Na}_{2} \mathrm{SO}_{4}$. The products were isolated by flash chromatography on silica gel (cyclohexane/diethyl ether $9 / 1$ as eluant).

Method B: To a stirred solution of $1(1 \mathrm{mmol})$ in $\mathrm{CH}_{2} \mathrm{Cl}_{2}(5 \mathrm{~mL}), \mathrm{N}$-bromosuccinimide $(0.5$ mmol, 0.5 equiv., $89 \mathrm{mg}$.) was added in one portion. The reaction was stirred at r.t. overnight and then quenched with water $(5 \mathrm{~mL})$. After dilution with $\mathrm{CH}_{2} \mathrm{Cl}_{2}(10 \mathrm{~mL})$, the organic layer was washed twice with water and dried over $\mathrm{Na}_{2} \mathrm{SO}_{4}$. The products were isolated by flash chromatography on silica gel (cyclohexane/diethyl ether $9 / 1$ as eluant).

(Z)-1-Benzyl-3-(2-bromoethylidene)-4-phenylazetidin-2-one (2a). ${ }^{1} \mathrm{H} \quad \mathrm{NMR} \quad(300 \mathrm{MHz}$, $\left.\mathrm{CDCl}_{3}\right) \delta_{\mathrm{H}} 3.92\left(1 \mathrm{H}, \mathrm{d}, J=15.0 \mathrm{~Hz}, \mathrm{CH}_{2} \mathrm{Ph}\right), 4.38\left(1 \mathrm{H}, \mathrm{dd}, J=8.7,10.5 \mathrm{~Hz}, C_{2} \mathrm{Br}\right), 4.47(1 \mathrm{H}, \mathrm{dd}$, $\left.J=8.7,10.5 \mathrm{~Hz}, \mathrm{CH}_{2} \mathrm{Br}\right), 4.84(1 \mathrm{H}, \mathrm{s}, \mathrm{CHPh}), 4.94\left(1 \mathrm{H}, \mathrm{d}, J=15.0 \mathrm{~Hz}, \mathrm{CH}_{2} \mathrm{Ph}\right), 5.71(1 \mathrm{H}, \mathrm{t}, J=8.7$ $\mathrm{Hz}, \mathrm{CH}=\mathrm{C}), 7.17-7.50(10 \mathrm{H}, \mathrm{m}, \mathrm{Ar}) ;{ }^{13} \mathrm{C} \mathrm{NMR}\left(75 \mathrm{MHz}, \mathrm{CDCl}_{3}\right) \delta_{\mathrm{C}} 26.1,44.3,61.6,124.1$, $127.4,127.8,128.5,128.8,128.9,129.0,135.0,135.8,145.2,162.8$; LC-ESI-MS rt 12.3 min., m/z 342-344 (M+1), 364-366 (M+Na); ); IR (film) 3065, 2913, 1739, 1706, 1428, 1315, 1262, $1070 \mathrm{~cm}^{-1}$; Calcd for $\mathrm{C}_{18} \mathrm{H}_{16}$ BrNO: C, 63.17; H, 4.71; N, 4.09. Found: C, 63.15; H, 4.70; N, 4.11. (E)-1-benzyl-3-(2-bromoethylidene)-4-phenylazetidin-2-one (2a). ${ }^{1} \mathrm{H} \mathrm{NMR}\left(300 \mathrm{MHz}, \mathrm{CDCl}_{3}\right)$ $\delta_{\mathrm{H}} 3.62\left(1 \mathrm{H}, \mathrm{dd}, J=8.4,10.5 \mathrm{~Hz}, \mathrm{CH}_{2} \mathrm{Br}\right), 3.65\left(1 \mathrm{H}, \mathrm{dd}, J=8.4,10.5 \mathrm{~Hz}, \mathrm{CH}_{2} \mathrm{Br}\right), 3.87(1 \mathrm{H}, \mathrm{d}$, $\left.J=15.0 \mathrm{~Hz}, \mathrm{CH}_{2} \mathrm{Ph}\right), 4.91\left(1 \mathrm{H}, \mathrm{d}, J=15.0 \mathrm{~Hz}, \mathrm{CH}_{2} \mathrm{Ph}\right), 4.96(1 \mathrm{H}, \mathrm{s}, C H \mathrm{Ph}), 6.39(1 \mathrm{H}, \mathrm{t}, J=8.4 \mathrm{~Hz}$, $\mathrm{CH}=\mathrm{C}), 7.05-7.10(2 \mathrm{H}, \mathrm{m}, \mathrm{Ar}), 7.10-7.50(8 \mathrm{H}, \mathrm{m}, \mathrm{Ar}) ;{ }^{13} \mathrm{C} \mathrm{NMR}\left(75 \mathrm{MHz}, \mathrm{CDCl}_{3}\right) \delta_{\mathrm{C}} 25.0,44.3$, $61.3,121.1,127.5,127.9,128.1,128.5,128.8,129.2$, 134.9, 138.8, 145.6, 163.0; LC-ESI-MS rt $11.8 \mathrm{~min} ., \mathrm{m} / \mathrm{z}$ 342-344 (M+1), 364-366 (M+Na); IR (film) 3066, 2910, 1733, 1429, 1315, 1261, $1077 \mathrm{~cm}^{-1}$; Calcd for $\mathrm{C}_{18} \mathrm{H}_{16}$ BrNO: C, 63.17; H, 4.71; N, 4.09. Found: C, 63.18; H, 4.74; N, 4.06. (Z)-1-benzyl-3-(2-bromobutylidene)-4-phenylazetidin-2-one (2b). First diastereomer: ${ }^{1} \mathrm{H}$ NMR $\left(300 \mathrm{MHz}, \mathrm{CDCl}_{3}\right) \delta_{\mathrm{H}} 1.00\left(3 \mathrm{H}, \mathrm{t}, J=7.8 \mathrm{~Hz}, \mathrm{CH}_{2} \mathrm{CH}_{3}\right), 1.77-2.05\left(2 \mathrm{H}, \mathrm{m}, \mathrm{CH}_{2} \mathrm{CH}_{3}\right), 3.87$ $\left(1 \mathrm{H}, \mathrm{d}, J=15.0 \mathrm{~Hz}, \mathrm{CH}_{2} \mathrm{Ph}\right), 4.81(1 \mathrm{H}, \mathrm{s}, C H \mathrm{Ph}), 4.88\left(1 \mathrm{H}, \mathrm{d}, J=15.0 \mathrm{~Hz}, C_{2} \mathrm{Ph}\right), 5.42(1 \mathrm{H}, \mathrm{dt}$, $J=7.4,10.8 \mathrm{~Hz}, C H B r), 5.61(1 \mathrm{H}, \mathrm{d}, J=10.8 \mathrm{~Hz}, C H=\mathrm{C}), 7.05-7.55(10 \mathrm{H}, \mathrm{m}, \mathrm{Ar})$; ${ }^{13} \mathrm{C} \mathrm{NMR}$ $\left(75 \mathrm{MHz}, \mathrm{CDCl}_{3}\right) \delta_{\mathrm{C}} 11.9,32.3,44.1,49.5,61.2,125.3,127.1,127.2,127.5,127.6,128.3,128.4$, 128.6, 128.7, 128.8, 128.9, 134.8, 141.2, 143.1, 162.6; LC-ESI-MS rt $17.5 \mathrm{~min} ., \mathrm{m} / \mathrm{z} 370-372$ $(\mathrm{M}+1), 392-394$ (M+Na); IR (film) 3031, 2968, 2931, 1748, 1655, 1496, 1456, 1381, 1353, 1288 $\mathrm{cm}^{-1}$; Calcd for $\mathrm{C}_{20} \mathrm{H}_{20} \mathrm{BrNO}$ : C, 64.87; H, 5.44; N, 3.78. Found: C, 64.86; H, 5.46; N, 3.80. 
Second diastereomer: ${ }^{1} \mathrm{H}$ NMR $\left(300 \mathrm{MHz}, \mathrm{CDCl}_{3}\right) \delta_{\mathrm{H}} 1.06\left(3 \mathrm{H}, \mathrm{t}, J=7.4 \mathrm{~Hz}, \mathrm{CH}_{2} \mathrm{CH}_{3}\right), 1.81-2.02$ $\left(2 \mathrm{H}, \mathrm{m}, \mathrm{CH}_{2} \mathrm{CH}_{3}\right), 3.86\left(1 \mathrm{H}, \mathrm{d}, \mathrm{J}=15.0 \mathrm{~Hz}, \mathrm{CH}_{2} \mathrm{Ph}\right), 4.82(1 \mathrm{H}, \mathrm{s}, \mathrm{CHPh}), 4.87(1 \mathrm{H}, \mathrm{d}, J=15.0 \mathrm{~Hz}$, $\left.\mathrm{CH}_{2} \mathrm{Ph}\right), 5.44(1 \mathrm{H}, \mathrm{dt}, \mathrm{J}=7.4,10.8 \mathrm{~Hz}, \mathrm{CHBr}), 5.64(1 \mathrm{H}, \mathrm{d}, J=10.8 \mathrm{~Hz}, \mathrm{CH}=\mathrm{C}), 7.05-7.60(10 \mathrm{H}$, $\mathrm{m}, \mathrm{Ar}) ;{ }^{13} \mathrm{C} \mathrm{NMR}\left(75 \mathrm{MHz}, \mathrm{CDCl}_{3}\right) \delta_{\mathrm{C}} 11.8,32.0,44.1,50.0,61.4,125.9,127.1,127.2,127.5$, 127.6, 128.3, 128.4, 128.6, 128.7, 128.8, 128.9, 135.0, 142.5, 144.0, 162.8; LC-ESI-MS rt 17.1 min., m/z 370-372 (M+1), 392-394 (M+Na); IR (film) 3032, 2967, 2931, 1748, 1654, 1496, 1455, 1380, $1070 \mathrm{~cm}^{-1}$; Calcd for $\mathrm{C}_{20} \mathrm{H}_{20} \mathrm{BrNO}$ : C, 64.87; H, 5.44; N, 3.78. Found: C, 64.85; H, $5.43 ; \mathrm{N}, 3.78$.

(E)-1-Benzyl-3-(2-bromobutylidene)-4-phenylazetidin-2-one (2b). First diastereomer: ${ }^{1} \mathrm{H}$ NMR $\left(300 \mathrm{MHz}, \mathrm{CDCl}_{3}\right) \delta_{\mathrm{H}} 0.53\left(3 \mathrm{H}, \mathrm{t}, J=7.2 \mathrm{~Hz}, \mathrm{CH}_{2} \mathrm{CH}_{3}\right), 1.50-1.75\left(2 \mathrm{H}, \mathrm{m}, \mathrm{CH}_{2} \mathrm{CH}_{3}\right), 3.87$ $\left(1 \mathrm{H}, \mathrm{d}, J=15.0 \mathrm{~Hz}, \mathrm{CH}_{2} \mathrm{Ph}\right), 4.10(1 \mathrm{H}, \mathrm{dt}, J=7.0,10.8 \mathrm{~Hz}, \mathrm{CHBr}), 4.88(1 \mathrm{H}, \mathrm{d}, J=15.0 \mathrm{~Hz}$, $\left.\mathrm{CH}_{2} \mathrm{Ph}\right), 4.95(1 \mathrm{H}, \mathrm{s}, \mathrm{CHPh}), 6.25(1 \mathrm{H}, \mathrm{d}, \mathrm{J}=10.8 \mathrm{~Hz}, \mathrm{CH}=\mathrm{C}), 7.00-7.50(10 \mathrm{H}, \mathrm{m}, \mathrm{Ar}) ;{ }^{13} \mathrm{C}$ NMR $\left(75 \mathrm{MHz}, \mathrm{CDCl}_{3}\right) \delta_{\mathrm{C}} 11.4,31.7,44.2,48.8,61.3,125.4,127.3,127.8,128.4,128.7,128.9,129.1$, 134.5, 134.9, 144.0, 163.0; LC-ESI-MS rt 16.4 min., m/z 370-372 (M+1), 392-394 (M+Na); ); IR (film) 3031, 2969, 2929, 1753, 1560, 1496, 1456, 1382, 1353, $1354 \mathrm{~cm}^{-1}$; Calcd for $\mathrm{C}_{20} \mathrm{H}_{20} \mathrm{BrNO}$ : C, 64.87; H, 5.44; N, 3.78. Found: C, 64.88; H, 5.42; N, 3.79. Second diastereomer: ${ }^{1} \mathrm{H}$ NMR $\left(300 \mathrm{MHz}, \mathrm{CDCl}_{3}\right) \delta_{\mathrm{H}} 0.93\left(3 \mathrm{H}, \mathrm{t}, J=7.4 \mathrm{~Hz}, \mathrm{CH}_{2} \mathrm{CH}_{3}\right), 1.77-1.90(2 \mathrm{H}, \mathrm{m}$, $\left.\mathrm{CH}_{2} \mathrm{CH}_{3}\right), 3.84\left(1 \mathrm{H}, \mathrm{d}, J=15.0 \mathrm{~Hz}, \mathrm{CH}_{2} \mathrm{Ph}\right), 4.01(1 \mathrm{H}, \mathrm{dt}, J=7.0,10.6 \mathrm{~Hz}, \mathrm{CHBr}), 4.86(1 \mathrm{H}, \mathrm{d}$, $\left.J=15.0 \mathrm{~Hz}, \mathrm{CH}_{2} \mathrm{Ph}\right), 4.87(1 \mathrm{H}, \mathrm{s}, \mathrm{CHPh}), 6.30(1 \mathrm{H}, \mathrm{d}, J=10.8 \mathrm{~Hz}, \mathrm{CH}=\mathrm{C}), 7.00-7.50$ (10H, m, $\mathrm{Ar}) ;{ }^{13} \mathrm{C} \mathrm{NMR}\left(75 \mathrm{MHz}, \mathrm{CDCl}_{3}\right) \delta_{\mathrm{C}} 11.9,31.7,44.2,49.3,61.5,125.5,127.3,127.8,128.4$, 128.7, 128.9, 129.0, 135.0, 135.7, 142.2, 163.0; LC-ESI-MS rt $15.3 \mathrm{~min} ., \mathrm{m} / \mathrm{z}$ 370-372 (M+1), 392-394 (M+Na); IR (film) 3031, 2967, 2927, 1751, 1685, 1676, 1495, 1456, $1354 \mathrm{~cm}^{-1}$; Calcd for $\mathrm{C}_{20} \mathrm{H}_{20}$ BrNO: C, 64.87; H, 5.44; N, 3.78. Found: C, 64.86; H, 5.44; N, 3.79.

Ethyl 3-((Z)-3-(2-bromobutylidene)-2-oxo-4-phenylazetidin-1-yl)propanoate (2c). First diastereomer: ${ }^{1} \mathrm{H}$ NMR $\left(300 \mathrm{MHz}, \mathrm{CDCl}_{3}\right) \delta_{\mathrm{H}} 1.04\left(3 \mathrm{H}, \mathrm{t}, J=7.2 \mathrm{~Hz}, \mathrm{CH}_{2} \mathrm{CH}_{3}\right), 1.22(3 \mathrm{H}, \mathrm{t}, J=7.2$ $\left.\mathrm{Hz}, \mathrm{COOCH}_{2} \mathrm{CH}_{3}\right), 1.80-2.05\left(2 \mathrm{H}, \mathrm{m}, \mathrm{CH}_{2} \mathrm{CH}_{3}\right), 2.50-2.80\left(2 \mathrm{H}, \mathrm{m}, \mathrm{CH}_{2} \mathrm{COOEt}\right), 3.32(1 \mathrm{H}, \mathrm{dt}$, $\left.J=6.8,14.4 \mathrm{~Hz}, \mathrm{NCH}_{2} \mathrm{CH}_{2}\right), 3.75\left(1 \mathrm{H}, \mathrm{dt}, J=6.8,14.4 \mathrm{~Hz}, \mathrm{NCH}_{2} \mathrm{CH}_{2}\right), 4.10(2 \mathrm{H}, \mathrm{q}, J=7.2 \mathrm{~Hz}$, $\left.\mathrm{COOCH}_{2} \mathrm{CH}_{3}\right), 5.00(1 \mathrm{H}, \mathrm{s}, \mathrm{CHPh}), 5.33(1 \mathrm{H}, \mathrm{dt}, J=7.0,11.0 \mathrm{~Hz}, \mathrm{CHBr}), 5.60(1 \mathrm{H}, \mathrm{d}, J=11.0$ $\mathrm{Hz}, \mathrm{CH}=\mathrm{C}), 7.20-7.50(5 \mathrm{H}, \mathrm{m}, \mathrm{Ar}) ;{ }^{13} \mathrm{C} \mathrm{NMR}\left(75 \mathrm{MHz}, \mathrm{CDCl}_{3}\right) \delta_{\mathrm{C}} 11.9,14.1,32.5,33.0,36.4$, 49.6, 60.9, 63.0, 125.4, 127.2, 128.9, 129.2, 135.6, 142.0, 163.2, 172.1; LC-ESI-MS rt 14.8 min., m/z 380-382 (M+1), 402-404 (M+Na); IR (film) 2940, 2923, 2853, 1700, 1456, 1419, 1402, 1377, 1305, 1200, $1173 \mathrm{~cm}^{-1}$; Calcd for $\mathrm{C}_{18} \mathrm{H}_{22} \mathrm{BrNO}_{3}$ : C, 56.85; H, 5.83; N, 3.68. Found: C, 56.87; H, 5.85; N, 3.71. Second diastereomer: ${ }^{1} \mathrm{H}$ NMR $\left(300 \mathrm{MHz}, \mathrm{CDCl}_{3}\right) \delta_{\mathrm{H}} 1.02(3 \mathrm{H}, \mathrm{t}, J=7.2$ $\left.\mathrm{Hz}, \mathrm{CH}_{2} \mathrm{CH}_{3}\right), 1.23\left(3 \mathrm{H}, \mathrm{t}, J=7.2 \mathrm{~Hz}, \mathrm{COOCH}_{2} \mathrm{CH}_{3}\right), 1.60-1.80\left(2 \mathrm{H}, \mathrm{m}, \mathrm{CH}_{2} \mathrm{CH}_{3}\right), 2.50-2.70(2 \mathrm{H}$, $\left.\mathrm{m}, \mathrm{CH}_{2} \mathrm{COOEt}\right), 3.35\left(1 \mathrm{H}, \mathrm{dt}, J=7.5,14.4 \mathrm{~Hz}, \mathrm{NCH}_{2} \mathrm{CH}_{2}\right), 3.76(1 \mathrm{H}, \mathrm{dt}, J=6.9,14.4 \mathrm{~Hz}$, $\left.\mathrm{NCH}_{2} \mathrm{CH}_{2}\right), 4.12\left(2 \mathrm{H}, \mathrm{q}, J=7.2 \mathrm{~Hz}, \mathrm{COOCH}_{2} \mathrm{CH}_{3}\right), 5.03(1 \mathrm{H}, \mathrm{s}, \mathrm{CHPh}), 5.37(1 \mathrm{H}, \mathrm{dt}, J=66,10.5$ $\mathrm{Hz}, \mathrm{CHBr}), 5.64(1 \mathrm{H}, \mathrm{d}, J=10.5 \mathrm{~Hz}, \mathrm{CH}=\mathrm{C}), 7.20-7.45(5 \mathrm{H}, \mathrm{m}, \mathrm{Ar}) ;{ }^{13} \mathrm{C} \mathrm{NMR}\left(75 \mathrm{MHz}, \mathrm{CDCl}_{3}\right)$ $\delta_{\mathrm{C}} 12.0,14.1,32.4,33.0,37.2,50.0,61.0,62.7,125.6,127.6,128.7,129.0,137.1,141.8,163.1$, 172.0; LC-ESI-MS rt 13.9 min., m/z 380-382 (M+1), 402-404 (M+Na); IR (film) 2924, 2854, 
1706, 1454, 1416, 1403, 1376, 1305, $1199 \mathrm{~cm}^{-1}$; Calcd for $\mathrm{C}_{18} \mathrm{H}_{22} \mathrm{BrNO}_{3}: \mathrm{C}, 56.85 ; \mathrm{H}, 5.83 ; \mathrm{N}$, 3.68. Found: C, 56.84; H, 5.82; N, 3.64.

Ethyl 3-((E)-3-(2-bromobutylidene)-2-oxo-4-phenylazetidin-1-yl)propanoate (2c). First diastereomer: ${ }^{1} \mathrm{H}$ NMR $\left(300 \mathrm{MHz}, \mathrm{CDCl}_{3}\right) \delta_{\mathrm{H}} 0.52\left(3 \mathrm{H}, \mathrm{t}, J=7.4 \mathrm{~Hz}, \mathrm{CH}_{2} \mathrm{CH}_{3}\right), 1.22(3 \mathrm{H}, \mathrm{t}, J=7.2$ $\left.\mathrm{Hz}, \mathrm{COOCH}_{2} \mathrm{CH}_{3}\right), 1.45-1.80\left(2 \mathrm{H}, \mathrm{m}, \mathrm{CH}_{2} \mathrm{CH}_{3}\right), 2.40-2.70\left(2 \mathrm{H}, \mathrm{m}, \mathrm{CH}_{2} \mathrm{COOEt}\right), 3.33(1 \mathrm{H}$, dt, $\left.J=7.0,14.6 \mathrm{~Hz}, \mathrm{NCH}_{2} \mathrm{CH}_{2}\right), 3.75\left(1 \mathrm{H}, \mathrm{dt}, J=6.6,14.6 \mathrm{~Hz}, \mathrm{NCH}_{2} \mathrm{CH}_{2}\right), 4.00-4.10(1 \mathrm{H}, \mathrm{m}, \mathrm{CHBr})$, $4.09\left(2 \mathrm{H}, \mathrm{q}, J=7.2 \mathrm{~Hz}, \mathrm{COOCH}_{2} \mathrm{CH}_{3}\right), 5.14\left(1 \mathrm{H}, \mathrm{d}, J_{1,3}=1.5 \mathrm{~Hz}, \mathrm{CHPh}\right), 6.21(1 \mathrm{H}, \mathrm{dd}, J=11.0 \mathrm{~Hz}$, $\left.J_{1,3}=1.5 \mathrm{~Hz}, \mathrm{CH}=\mathrm{C}\right), 7.25-7.45(5 \mathrm{H}, \mathrm{m}, \mathrm{Ar}) ;{ }^{13} \mathrm{C} \mathrm{NMR}\left(75 \mathrm{MHz}, \mathrm{CDCl}_{3}\right) \delta_{\mathrm{C}} 11.5,14.0,31.8$, 33.1, 36.4, 48.8, 60.8, 62.7, 125.5, 127.3, 129.1, 129.7, 133.5, 144.1, 163.5, 171.0; LC-ESI-MS rt 14.2 min., m/z 380-382 (M+1), 402-404 (M+Na); IR (film) 2972, 2932, 1734, 1457, 1376, 1189, 1112, 1071, $1020 \mathrm{~cm}^{-1}$; Calcd for $\mathrm{C}_{18} \mathrm{H}_{22} \mathrm{BrNO}_{3}$ : C, 56.85; H, 5.83; N, 3.68. Found: C, 56.83; H, 5.81; N, 3.70. Second diastereomer: ${ }^{1} \mathrm{H}$ NMR $\left(300 \mathrm{MHz}, \mathrm{CDCl}_{3}\right) \delta_{\mathrm{H}} 0.96(3 \mathrm{H}, \mathrm{t}, J=7.4$ $\left.\mathrm{Hz}, \mathrm{CH}_{2} \mathrm{CH}_{3}\right), 1.23\left(3 \mathrm{H}, \mathrm{t}, J=7.2 \mathrm{~Hz}, \mathrm{COOCH}_{2} \mathrm{CH}_{3}\right), 1.81-1.96\left(2 \mathrm{H}, \mathrm{m}, \mathrm{CH}_{2} \mathrm{CH}_{3}\right), 2.40-2.80(2 \mathrm{H}$, $\left.\mathrm{m}, \mathrm{CH}_{2} \mathrm{COOEt}\right), 3.34\left(1 \mathrm{H}, \mathrm{dt}, \mathrm{J}=6.6,14.8 \mathrm{~Hz}, \mathrm{NCH}_{2} \mathrm{CH}_{2}\right), 3.71(1 \mathrm{H}, \mathrm{dt}, J=6.2,14.8 \mathrm{~Hz}$, $\left.\mathrm{NCH}_{2} \mathrm{CH}_{2}\right), 3.96-4.10(1 \mathrm{H}, \mathrm{m}, \mathrm{CHBr}), 4.09\left(2 \mathrm{H}, \mathrm{q}, J=7.2 \mathrm{~Hz}, \mathrm{COOCH}_{2} \mathrm{CH}_{3}\right), 5.08(1 \mathrm{H}, \mathrm{s}$, CHPh), $6.28(1 \mathrm{H}, \mathrm{dd}, J=11.0 \mathrm{~Hz}, \mathrm{CH}=\mathrm{C}), 7.25-7.45(5 \mathrm{H}, \mathrm{m}, \mathrm{Ar}) ;{ }^{13} \mathrm{C} \mathrm{NMR}\left(75 \mathrm{MHz}, \mathrm{CDCl}_{3}\right) \delta_{\mathrm{C}}$ 12.0, 14.1, 31.8, 33.1, 36.4, 49.2, 60.9, 63.1, 125.6, 127.8, 129.1, 130.2, 135.0, 142.2, 163.6, 171.2; LC-ESI-MS rt 13.7 min., m/z 380-382 (M+1), 402-404 (M+Na); IR (film) 2985, 2928, 1734, 1457, 1376, $1243 \mathrm{~cm}^{-1}$; Calcd for $\mathrm{C}_{18} \mathrm{H}_{22} \mathrm{BrNO}_{3}$ : C, 56.85; H, 5.83; N, 3.68. Found: C, 56.82; H, 5.87; N, 3.72 .

(Z)-1-Benzyl-3-(2-bromobutylidene)-4-(4-nitrophenyl)azetidin-2-one $\quad$ (2d). First diastereomer: ${ }^{1} \mathrm{H}$ NMR $\left(300 \mathrm{MHz}, \mathrm{CDCl}_{3}\right) \delta_{\mathrm{H}} 0.98\left(3 \mathrm{H}, \mathrm{t}, J=7.4 \mathrm{~Hz}, \mathrm{CH}_{2} \mathrm{CH}_{3}\right), 1.76-2.05(2 \mathrm{H}, \mathrm{m}$, $\left.\mathrm{CH}_{2} \mathrm{CH}_{3}\right), 4.02\left(1 \mathrm{H}, \mathrm{d}, \mathrm{J}=15.0 \mathrm{~Hz}, \mathrm{CH}_{2} \mathrm{Ph}\right), 4.86\left(1 \mathrm{H}, \mathrm{d}, \mathrm{J}=15.0 \mathrm{~Hz}, \mathrm{CH}_{2} \mathrm{Ph}\right), 4.90(1 \mathrm{H}, \mathrm{s}, \mathrm{CHPh})$, $5.32(1 \mathrm{H}, \mathrm{dt}, J=7.4,10.6 \mathrm{~Hz}, C H \mathrm{Br}), 5.63(1 \mathrm{H}, \mathrm{d}, J=10.6 \mathrm{~Hz}, C H=\mathrm{C}), 7.00-7.20$ (2H, m, Ar), 7.20-7.40 (5H, m, Ar), $8.20(2 \mathrm{H}, \mathrm{d}, J=7.8 \mathrm{~Hz}, \mathrm{Ar}) ;{ }^{13} \mathrm{C} \mathrm{NMR}\left(75 \mathrm{MHz}, \mathrm{CDCl}_{3}\right) \delta_{\mathrm{C}} 12.0,32.2$, 44.9, 49.1, 60.7, 124.1, 127.9, 128.1, 128.5, 128.9, 129.7, 134.3, 142.3, 143.8, 148.1, 162.4; LCESI-MS rt 15.8 min., m/z 415-417 (M+1), 437-439 (M+Na); IR (film) 3066, 2969, 2932, 1751, 1603, 1522, 1496, 1349, 1291, 1106, $1072 \mathrm{~cm}^{-1}$; Calcd for $\mathrm{C}_{20} \mathrm{H}_{19} \mathrm{BrN}_{2} \mathrm{O}_{3}: \mathrm{C}, 57.84 ; \mathrm{H}, 4.61$; , 6.75. Found: C, 57.80; H, 4.58; N, 6.78. Second diastereomer: ${ }^{1} \mathrm{H}$ NMR $\left(300 \mathrm{MHz}, \mathrm{CDCl}_{3}\right) \delta_{\mathrm{H}}$ $1.05\left(3 \mathrm{H}, \mathrm{t}, J=7.4 \mathrm{~Hz}, \mathrm{CH}_{2} \mathrm{CH}_{3}\right), 1.86-2.05\left(2 \mathrm{H}, \mathrm{m}, \mathrm{CH}_{2} \mathrm{CH}_{3}\right), 3.98\left(1 \mathrm{H}, \mathrm{d}, J=15.0 \mathrm{~Hz}, \mathrm{CH}_{2} \mathrm{Ph}\right)$, $4.84\left(1 \mathrm{H}, \mathrm{d}, J=15.0 \mathrm{~Hz}, \mathrm{CH}_{2} \mathrm{Ph}\right), 4.88(1 \mathrm{H}, \mathrm{s}, \mathrm{CHPh}), 5.37(1 \mathrm{H}, \mathrm{dt}, J=6.6,10.6 \mathrm{~Hz}, \mathrm{CHBr}), 5.64$ (1H, d, J=10.6 Hz, $C H=C), 7.11-7.14$ (2H, m, Ar), 7.28-7.47 (5H, m, Ar), 8.18-8.24 (2H, m, Ar); ${ }^{13} \mathrm{C}$ NMR $\left(75 \mathrm{MHz}, \mathrm{CDCl}_{3}\right) \quad \delta_{\mathrm{C}} 11.8,32.0,44.8,49.6,60.4,124.1,126.4,128.5,128.9,129.8$, 134.3, 141.6, 143.6, 148.1, 162.6; LC-ESI-MS rt 15.5 min., m/z 415-417 (M+1), 437-439 $(\mathrm{M}+\mathrm{Na})$; IR (film) 3061, 3029, 2968, 2931, 1752, 1654, 1522, $1349 \mathrm{~cm}^{-1}$; Calcd for $\mathrm{C}_{20} \mathrm{H}_{19} \mathrm{BrN}_{2} \mathrm{O}_{3}$ : C, 57.84; H, 4.61; N, 6.75. Found: C, 57.85; H, 4.59; N, 6.74.

(E)-1-Benzyl-3-(2-bromobutylidene)-4-(4-nitrophenyl)azetidin-2-one $\quad$ (2d). First diastereomer: ${ }^{1} \mathrm{H}$ NMR $\left(300 \mathrm{MHz}, \mathrm{CDCl}_{3}\right) \delta_{\mathrm{H}} 0.57\left(3 \mathrm{H}, \mathrm{t}, J=7.4 \mathrm{~Hz}, \mathrm{CH}_{2} \mathrm{CH}_{3}\right), 1.57-1.77(2 \mathrm{H}, \mathrm{m}$, $\left.\mathrm{CH}_{2} \mathrm{CH}_{3}\right), 3.98\left(1 \mathrm{H}, \mathrm{d}, \mathrm{J}=15.0 \mathrm{~Hz}, \mathrm{CH}_{2} \mathrm{Ph}\right), 4.05(1 \mathrm{H}, \mathrm{m}, \mathrm{CHBr}), 4.84(1 \mathrm{H}, \mathrm{d}, J=15.0 \mathrm{~Hz}$, $\left.\mathrm{CH}_{2} \mathrm{Ph}\right), 5.03(1 \mathrm{H}, \mathrm{s}, \mathrm{CHPh}), 6.32(1 \mathrm{H}, \mathrm{d}, \mathrm{J}=10.8 \mathrm{~Hz}, \mathrm{CH}=\mathrm{C}), 7.12-7.14$ (2H, m, Ar), 7.28-7.46 
(5H, m, Ar), $8.20(2 \mathrm{H}, \mathrm{m}, \mathrm{Ar}) ;{ }^{13} \mathrm{C}$ NMR $\left(75 \mathrm{MHz}, \mathrm{CDCl}_{3}\right) \delta_{\mathrm{C}} 11.6,31.6,44.9,48.0,60.5,124.2$, 126.4, 128.3, 128.8, 129.8, 134.5, 141.6, 143.3, 148.1, 162.4; LC-ESI-MS rt 14.6 min., m/z 415417 (M+1), 437-439 (M+Na); IR (film) 3065, 2970, 2927, 1752, 1656, 1607, 1522, $1349 \mathrm{~cm}^{-1}$; Calcd for $\mathrm{C}_{20} \mathrm{H}_{19} \mathrm{BrN}_{2} \mathrm{O}_{3}$ : C, 57.84; H, 4.61; N, 6.75. Found: C, 57.81; H, 4.63; N, 6.74. Second diastereomer: ${ }^{1} \mathrm{H}$ NMR $\left(300 \mathrm{MHz}, \mathrm{CDCl}_{3}\right) \delta_{\mathrm{H}} 0.94\left(3 \mathrm{H}, \mathrm{t}, J=7.4 \mathrm{~Hz}, \mathrm{CH}_{2} \mathrm{CH}_{3}\right), 1.78-2.05(2 \mathrm{H}, \mathrm{m}$, $\left.\mathrm{CH}_{2} \mathrm{CH}_{3}\right), 3.89-3.97(1 \mathrm{H}, \mathrm{m}, \mathrm{CHBr}), 3.98\left(1 \mathrm{H}, \mathrm{d}, \mathrm{J}=15.0 \mathrm{~Hz}, \mathrm{CH}_{2} \mathrm{Ph}\right), 4.84(1 \mathrm{H}, \mathrm{d}, \mathrm{J}=15.0 \mathrm{~Hz}$, $\left.\mathrm{CH}_{2} \mathrm{Ph}\right), 4.96(1 \mathrm{H}, \mathrm{s}, \mathrm{CHPh}), 6.35(1 \mathrm{H}, \mathrm{d}, \mathrm{J}=11.0 \mathrm{~Hz}, \mathrm{CH}=\mathrm{C}), 7.10-7.15(2 \mathrm{H}, \mathrm{m}, \mathrm{Ar}), 7.28-7.47$ (5H, m, Ar), 8.21-8.32 (2H, m, Ar); ${ }^{13} \mathrm{C}$ NMR $\left(75 \mathrm{MHz}, \mathrm{CDCl}_{3}\right) \delta_{\mathrm{C}} 11.9,31.6,44.8,48.9,60.6$, $124.2,126.5,128.1,128.5,128.8,128.9,134.4,141.5,142.4,148.3,162.6$; LC-ESI-MS rt $14.2 \mathrm{~min} ., \mathrm{m} / \mathrm{z}$ 415-417 (M+1), 437-439 (M+Na); IR (film) 3061, 2970, 2933, 1751, 1606, 1521, $1345 \mathrm{~cm}^{-1}$; Calcd for $\mathrm{C}_{20} \mathrm{H}_{19} \mathrm{BrN}_{2} \mathrm{O}_{3}: \mathrm{C}, 57.84 ; \mathrm{H}, 4.61 ; \mathrm{N}, 6.75$. Found: $\mathrm{C}, 57.83 ; \mathrm{H}, 4.61 ; \mathrm{N}$, 6.77.

(Z)-1-(1'S-Phenylethyl)-3-(2-bromobutylidene)-4-(4-nitrophenyl)azetidin-2-one (2e). First diastereomer: ${ }^{1} \mathrm{H}$ NMR $\left(300 \mathrm{MHz}, \mathrm{CDCl}_{3}\right) \delta_{\mathrm{H}} 1.00\left(3 \mathrm{H}, \mathrm{t}, J=7.4 \mathrm{~Hz}, \mathrm{CH}_{2} \mathrm{CH}_{3}\right), 1.32(1 \mathrm{H}, \mathrm{d}$, $\left.J=7.4 \mathrm{~Hz}, \mathrm{CHCH}_{3}\right), 1.80-1.95\left(2 \mathrm{H}, \mathrm{m}, \mathrm{CH}_{2} \mathrm{CH}_{3}\right), 4.68(1 \mathrm{H}, \mathrm{bs}, \mathrm{CHPh}), 5.11(1 \mathrm{H}, \mathrm{q}, J=7.4 \mathrm{~Hz}$, $\left.\mathrm{CHCH}_{3}\right), 5.35(1 \mathrm{H}, \mathrm{m}, \mathrm{CHBr}) 5.53(1 \mathrm{H}, \mathrm{d}, \mathrm{J}=11.8 \mathrm{~Hz}, \mathrm{CH}=\mathrm{C}), 7.21-7.39(10 \mathrm{H}, \mathrm{m}, \mathrm{Ar}),{ }^{13} \mathrm{C}$ NMR $\left(75 \mathrm{MHz}, \mathrm{CDCl}_{3}\right) \delta_{\mathrm{C}} 12.0,32.2,44.9,49.1,60.7,124.1,127.9,128.1,128.5,128.9,129.7$, 134.3, 142.3, 143.8, 148.1, 162.4; LC-ESI-MS rt $15.2 \mathrm{~min} ., \mathrm{m} / \mathrm{z}$ 384-386 (M+1), 406-408 (M+Na); IR (film) 3066, 2969, 2932, 1751, 1603, 1522, 1496, 1349, 1291, 1106, $1072 \mathrm{~cm}^{-1}$; Calcd for $\mathrm{C}_{21} \mathrm{H}_{22} \mathrm{BrNO}$ : C, 65.63; H, 5.77; N, 3.64. Found: C, 65.61; H, 5.75; N, 3.62. Second diastereomer: ${ }^{1} \mathrm{H}$ NMR $\left(300 \mathrm{MHz}, \mathrm{CDCl}_{3}\right) \delta_{\mathrm{H}} 1.03\left(3 \mathrm{H}, \mathrm{t}, J=7.4 \mathrm{~Hz}, \mathrm{CH}_{2} \mathrm{CH}_{3}\right), 1.22(1 \mathrm{H}, \mathrm{d}, J=7.4$ $\left.\mathrm{Hz}, \mathrm{CHCH}_{3}\right), 1.88-2.00\left(2 \mathrm{H}, \mathrm{m}, \mathrm{CH}_{2} \mathrm{CH}_{3}\right), 4.61(1 \mathrm{H}, \mathrm{bs}, \mathrm{CHPh}), 5.05\left(1 \mathrm{H}, \mathrm{q}, J=7.4 \mathrm{~Hz}, \mathrm{CHCH}_{3}\right)$, $5.39(1 \mathrm{H}, \mathrm{m}, \mathrm{CHBr}) 5.52(1 \mathrm{H}, \mathrm{d}, J=11.5 \mathrm{~Hz}, \mathrm{CH}=\mathrm{C}), 7.19-7.45(10 \mathrm{H}, \mathrm{m}, \mathrm{Ar}),{ }^{13} \mathrm{C}$ NMR $\left(75 \mathrm{MHz}, \mathrm{CDCl}_{3}\right) \delta_{\mathrm{C}} 11.8,32.0,44.8,49.6,60.4,124.1,126.4,128.5,128.9,129.8,134.3,141.6$, 143.6, 148.1, 162.6; LC-ESI-MS rt $14.4 \mathrm{~min} ., \mathrm{m} / \mathrm{z}$ 384-386 (M+1), 406-408 (M+Na); IR (film) $3061,3029,2968,2931,1752,1654,1522,1349 \mathrm{~cm}^{-1}$; Calcd for $\mathrm{C}_{21} \mathrm{H}_{22} \mathrm{BrNO}$ : C, 65.63; H, 5.77; N, 3.64. Found: C, 65.60; H, 5.78; N, 3.64 .

(E)-1-(1'S-Phenylethyl)-3-(2-bromobutylidene)-4-(4-nitrophenyl)azetidin-2-one (2e).

First diastereomer: ${ }^{1} \mathrm{H}$ NMR $\left(300 \mathrm{MHz}, \mathrm{CDCl}_{3}\right) \delta_{\mathrm{H}} 0.53\left(3 \mathrm{H}, \mathrm{t}, J=7.4 \mathrm{~Hz}, \mathrm{CH}_{2} \mathrm{CH}_{3}\right), 1.34(1 \mathrm{H}, \mathrm{d}$, $\left.J=7.4 \mathrm{~Hz}, \mathrm{CHCH}_{3}\right), 1.42-1.70\left(2 \mathrm{H}, \mathrm{m}, \mathrm{CH}_{2} \mathrm{CH}_{3}\right), 3.99(1 \mathrm{H}, \mathrm{dt}, J=10.5,16.3 \mathrm{~Hz}, \mathrm{CHBr}), 4.82$ $(1 \mathrm{H}, \mathrm{bs}, \mathrm{CHPh}), 5.15\left(1 \mathrm{H}, \mathrm{q}, J=7.4 \mathrm{~Hz}, \mathrm{CHCH}_{3}\right), 6.20(1 \mathrm{H}, \mathrm{d}, J=10.5 \mathrm{~Hz}, \mathrm{CH}=\mathrm{C}), 7.22-7.40$ $(10 \mathrm{H}, \mathrm{m}, \mathrm{Ar}),{ }^{13} \mathrm{C} \mathrm{NMR}\left(75 \mathrm{MHz}, \mathrm{CDCl}_{3}\right) \delta_{\mathrm{C}} 11.6,31.6,44.9,48.0,60.5,124.2,126.4,128.3$, 128.8, 129.8, 134.5, 141.6, 143.3, 148.1, 162.4; LC-ESI-MS rt 15.3 min., m/z 384-386 (M+1), 406-408 (M+Na); IR (film) 3065, 2970, 2927, 1752, 1656, 1607, 1522, $1349 \mathrm{~cm}^{-1}$; Calcd for $\mathrm{C}_{21} \mathrm{H}_{22} \mathrm{BrNO}: \mathrm{C}$, 65.63; H, 5.77; N, 3.64. Found: $\mathrm{C}, 65.63 ; \mathrm{H}, 5.79 ; \mathrm{N}, 3.66$. Second diastereomer: ${ }^{1} \mathrm{H}$ NMR $\left(300 \mathrm{MHz}, \mathrm{CDCl}_{3}\right) \delta_{\mathrm{H}} 0.98\left(3 \mathrm{H}, \mathrm{t}, J=7.4 \mathrm{~Hz}, \mathrm{CH}_{2} \mathrm{CH}_{3}\right), 1.30(1 \mathrm{H}, \mathrm{d}, J=7.4$ $\left.\mathrm{Hz}, \mathrm{CHCH}_{3}\right), 1.40-1.60\left(2 \mathrm{H}, \mathrm{m}, \mathrm{CH}_{2} \mathrm{CH}_{3}\right), 3.90(1 \mathrm{H}, \mathrm{dt}, \mathrm{J}=11.1,6.9 \mathrm{~Hz}, \mathrm{CHBr}), 4.74(1 \mathrm{H}, \mathrm{bs}$, $C H P h), 5.17\left(1 \mathrm{H}, \mathrm{q}, J=7.4 \mathrm{~Hz}, \mathrm{CHCH}_{3}\right), 6.28(1 \mathrm{H}, \mathrm{d}, J=11.1 \mathrm{~Hz}, \mathrm{CH}=\mathrm{C}), 7.20-7.45(10 \mathrm{H}, \mathrm{m}$, $\mathrm{Ar}),{ }^{13} \mathrm{C}$ NMR $\left(75 \mathrm{MHz}, \mathrm{CDCl}_{3}\right) \quad \delta_{\mathrm{C}} 11.9,31.6,44.8,48.9,60.6,124.2,126.5,128.1,128.5$, 128.8, 128.9, 134.4, 141.5, 142.4, 148.3, 162.6; LC-ESI-MS rt 15.8 min., m/z 384-386 (M+1), 
406-408 (M+Na); IR (film) 3061, 2970, 2933, 1751, 1606, 1521, $1345 \mathrm{~cm}^{-1}$; Calcd for $\mathrm{C}_{21} \mathrm{H}_{22} \mathrm{BrNO}$ : C, 65.63; H, 5.77; N, 3.64. Found: C, 65.61; H, 5.74; N, 3.67.

\section{General procedure for the formation of esters $\mathrm{Z} / \mathrm{E}-3$ and $\mathrm{Z} / \mathrm{E}-4$}

Bromoderivative 2 ( $1 \mathrm{mmol})$ and resin supported benzoic acid or phenylacetic acid ( $3 \mathrm{mmol}, 3$ equiv., $1.43 \mathrm{~g}$ of resin with $2.1 \mathrm{mmol} / \mathrm{g}$ loading) were stirred in refluxing hexane for three hours. The resin was then separated by filtration and solvent removed under reduced pressure. The products of the reaction were purified by flash chromatography on silica gel (cyclohexane/ethyl acetate $8 / 2$ as eluant).

(Z)-2-(1-Benzyl-2-oxo-4-phenylazetidin-3-ylidene)ethyl benzoate (3a). ${ }^{1} \mathrm{H}$ NMR (300MHz, $\left.\mathrm{CDCl}_{3}\right) \delta_{\mathrm{H}} 3.92\left(1 \mathrm{H}, \mathrm{d}, J=15.0 \mathrm{~Hz}, \mathrm{CH}_{2} \mathrm{Ph}\right), 4.83(1 \mathrm{H}, \mathrm{s}, C H \mathrm{Ph}), 4.95(1 \mathrm{H}, \mathrm{d}, J=15.0 \mathrm{~Hz}$, $\left.\mathrm{CH}_{2} \mathrm{Ph}\right), 5.25\left(1 \mathrm{H}, \mathrm{dd}, \mathrm{J}=6.6,14.0 \mathrm{~Hz}, \mathrm{CH}_{2} \mathrm{OCOPh}\right), 5.32\left(1 \mathrm{H}, \mathrm{dd}, \mathrm{J}=6.6,14.0 \mathrm{~Hz}, \mathrm{CH}_{2} \mathrm{OCOPh}\right)$, $5.71(1 \mathrm{H}, \mathrm{t}, J=6.6 \mathrm{~Hz}, \mathrm{CH}=\mathrm{C}), 7.05-7.50(15 \mathrm{H}, \mathrm{m}, \mathrm{Ar}) ;{ }^{13} \mathrm{C} \mathrm{NMR}\left(75 \mathrm{MHz}, \mathrm{CDCl}_{3}\right) \delta_{\mathrm{C}} 44.3$, $60.6,61.6,122.7,127.3,127.8,128.3,128.4,128.5,128.7,128.8,128.9,129.6,133.0,135.1$, 135.9, 146.0, 163.0, 166.2; LC-ESI-MS rt 12.8 min., m/z 384 (M+1), 406 (M+Na); IR (film) 2966, 2918, 2846, 1766, 1455, 1375, 1260, 1102, $1001 \mathrm{~cm}^{-1}$; Calcd for $\mathrm{C}_{25} \mathrm{H}_{21} \mathrm{NO}_{3}$ : C, 78.31; H, 5.52; N, 3.65. Found: C, 78.32; H, 5.55; N, 3.64.

(E)-2-(1-Benzyl-2-oxo-4-phenylazetidin-3-ylidene)ethyl benzoate (3a). ${ }^{1} \mathrm{H}$ NMR (300MHz, $\left.\mathrm{CDCl}_{3}\right) \delta_{\mathrm{H}} 3.85\left(1 \mathrm{H}, \mathrm{d}, J=15.0 \mathrm{~Hz}, \mathrm{CH}_{2} \mathrm{Ph}\right), 4.57\left(1 \mathrm{H}, \mathrm{dd}, J=6.0,14.0 \mathrm{~Hz}, \mathrm{CH}_{2} \mathrm{OCOPh}\right), 4.91$ $\left(1 \mathrm{H}, \mathrm{dd}, J=6.0,14.0 \mathrm{~Hz}, \mathrm{CH}_{2} \mathrm{OCOPh}\right), 4.91\left(1 \mathrm{H}, \mathrm{d}, J=15.0 \mathrm{~Hz}, \mathrm{CH}_{2} \mathrm{Ph}\right), 5.02(1 \mathrm{H}, \mathrm{s}, \mathrm{CHPh})$, $6.40(1 \mathrm{H}, \mathrm{t}, J=6.0 \mathrm{~Hz}, \mathrm{CH}=\mathrm{C}), 7.05-7.50(15 \mathrm{H}, \mathrm{m}, \mathrm{Ar}) ;{ }^{13} \mathrm{C} \mathrm{NMR}\left(75 \mathrm{MHz}, \mathrm{CDCl}_{3}\right) \delta_{\mathrm{C}} 44.2$, 60.3 , 62.0, 119.6, 127.6, 127.7, 128.2, 128.3, 128.4, 128.7, 129.0, 129.5, 130.1, 133.4, 135.0, 135.3, 145.5, 163.3, 165.8; LC-ESI-MS rt 12.4 min., m/z 384 (M+1), 406 (M+Na); IR (film) 2966, 2919, 2840, 1759, 1713, 1454, 1375, 1250, 1103, $1010 \mathrm{~cm}^{-1}$; Calcd for $\mathrm{C}_{25} \mathrm{H}_{21} \mathrm{NO}_{3}$ : C, 78.31; H, 5.52; N, 3.65. Found: C, 78.33; H, 5.54; N, 3.64.

(Z)-1-(1-Benzyl-2-oxo-4-phenylazetidin-3-ylidene)butan-2-yl benzoate (3b). First diastereomer: ${ }^{1} \mathrm{H}$ NMR $\left(300 \mathrm{MHz}, \mathrm{CDCl}_{3}\right) \delta_{\mathrm{H}} 1.08\left(3 \mathrm{H}, \mathrm{t}, J=7.5 \mathrm{~Hz}, \mathrm{CH}_{2} \mathrm{CH}_{3}\right), 1.85-2.00(2 \mathrm{H}, \mathrm{m}$, $\left.\mathrm{CH}_{2} \mathrm{CH}_{3}\right), 3.90\left(1 \mathrm{H}, \mathrm{d}, J=15.0 \mathrm{~Hz}, \mathrm{CH}_{2} \mathrm{Ph}\right), 4.82(1 \mathrm{H}, \mathrm{bs}, \mathrm{CHPh}), 4.93(1 \mathrm{H}, \mathrm{d}, \mathrm{J}=15.0 \mathrm{~Hz}$, $\left.\mathrm{CH}_{2} \mathrm{Ph}\right), 5.54(1 \mathrm{H}, \mathrm{d}, J=8.1 \mathrm{~Hz}, \mathrm{CH}=\mathrm{C}), 6.15(1 \mathrm{H}, \mathrm{dt}, J=8.1,6.6 \mathrm{~Hz}, \mathrm{CHOCOPh}), 7.20-7.49$ $(13 \mathrm{H}, \mathrm{m}, \mathrm{Ar}), 8.07-8.11(2 \mathrm{H}, \mathrm{m}, \mathrm{Ar}) ;{ }^{13} \mathrm{C} \mathrm{NMR}\left(75 \mathrm{MHz}, \mathrm{CDCl}_{3}\right) \delta_{\mathrm{C}} 9.3,27.8,44.2,61.5,72.6$, $127.1,127.3,127.4,127.7,128.2,128.5,128.7,128.8,129.0,129.6,132.8,135.1,144.8,162.6$, 168.4; LC-ESI-MS rt $19.2 \mathrm{~min} ., \mathrm{m} / \mathrm{z} 412(\mathrm{M}+1), 434(\mathrm{M}+\mathrm{Na}), 845$ (2M+Na); IR (film): 3063, 2930, 2876, 1751, 1674, 1455, $1269 \mathrm{~cm}^{-1}$; Calcd for $\mathrm{C}_{27} \mathrm{H}_{25} \mathrm{NO}_{3}$ : C, 78.81; H, 6.12; N, 3.40. Found: C, 78.83; H, 6.11; N, 3.44. Second diastereomer: ${ }^{1} \mathrm{H}$ NMR $\left(300 \mathrm{MHz}, \mathrm{CDCl}_{3}\right) \delta_{\mathrm{H}} 0.75$ $\left(3 \mathrm{H}, \mathrm{t}, J=7.4 \mathrm{~Hz}, \mathrm{CH}_{2} \mathrm{CH}_{3}\right), 1.80-1.95\left(2 \mathrm{H}, \mathrm{m}, \mathrm{CH}_{2} \mathrm{CH}_{3}\right), 3.91\left(1 \mathrm{H}, \mathrm{d}, J=15.0 \mathrm{~Hz}, \mathrm{CH}_{2} \mathrm{Ph}\right), 4.84$ $(1 \mathrm{H}, \mathrm{bs}, C H \mathrm{Ph}), 4.92\left(1 \mathrm{H}, \mathrm{d}, J=15.0 \mathrm{~Hz}, \mathrm{CH}_{2} \mathrm{Ph}\right), 5.44(1 \mathrm{H}, \mathrm{m}, C H O C O P h), 5.70(1 \mathrm{H}, \mathrm{d}, J=10.4$ $\mathrm{Hz}, \mathrm{CH}=\mathrm{C}), 7.16-7.46(15 \mathrm{H}, \mathrm{m}, \mathrm{Ar}),{ }^{13} \mathrm{C} \mathrm{NMR}\left(75 \mathrm{MHz}, \mathrm{CDCl}_{3}\right) \delta_{\mathrm{C}} 9.6,26.5,44.2,61.3,72.5$, $126.1,127.3,127.6,127.7,127.9,128.2,128.5,128.7,128.8,128.9,133.9,134.9,143.2,162.8$, 168.6; LC-ESI-MS rt 17.6 min., m/z $412(\mathrm{M}+1), 434(\mathrm{M}+\mathrm{Na}), 845$ (2M+Na); IR (film): 2956, 
2925, 28726, 1751, 1654, 1457, $1380 \mathrm{~cm}^{-1}$; Calcd for $\mathrm{C}_{27} \mathrm{H}_{25} \mathrm{NO}_{3}: \mathrm{C}, 78.81 ; \mathrm{H}, 6.12 ; \mathrm{N}, 3.40$. Found: C, 78.79; H, 6.10; N, 3.41.

(E)-1-(1-Benzyl-2-oxo-4-phenylazetidin-3-ylidene)butan-2-yl benzoate (3b). First diastereomer: ${ }^{1} \mathrm{H}$ NMR $\left(300 \mathrm{MHz}, \mathrm{CDCl}_{3}\right) \delta_{\mathrm{H}} 0.91\left(3 \mathrm{H}, \mathrm{t}, J=7.5 \mathrm{~Hz}, \mathrm{CH}_{2} \mathrm{CH}_{3}\right), 1.58-1.80(2 \mathrm{H}, \mathrm{m}$, $\left.\mathrm{CH}_{2} \mathrm{CH}_{3}\right), 3.78\left(1 \mathrm{H}, \mathrm{d}, J=15.0 \mathrm{~Hz}, \mathrm{CH}_{2} \mathrm{Ph}\right), 4.83\left(1 \mathrm{H}, \mathrm{d}, J=15.0 \mathrm{~Hz}, \mathrm{CH}_{2} \mathrm{Ph}\right), 4.84(1 \mathrm{H}, \mathrm{bs}$, CHPh), 5.34 (1H, q, J=6.3 Hz, CHOCOPh), 6.37 (1H, dd, J=6.3 Hz, $\left.J_{1,3}=1.5 \mathrm{~Hz}, C H=\mathrm{C}\right), 7.13-$ 7.19 (4H, m, Ar), 7.27-7.40 (8H, m, Ar), 7.55 (1H, t, J=7.5 Hz, Ar), 7.76 (2H, t, J=8.4 Hz, Ar); ${ }^{13} \mathrm{C}$ NMR $\left(75 \mathrm{MHz}, \mathrm{CDCl}_{3}\right) \quad \delta_{\mathrm{C}} 9.4,27.4,44.1,62.5,72.3,124.3,127.7,128.0,128.2,128.4$, 128.7, 128.8, 128.9, 129.6, 129.7, 132.9, 135.3, 143.2, 163.7, 165.3; LC-ESI-MS rt 13.0 min., m/z $412(\mathrm{M}+1), 434(\mathrm{M}+\mathrm{Na})$; IR (film): 3061, 2929, 1712, 1656, 1455, $1270 \mathrm{~cm}^{-1}$; Calcd for $\mathrm{C}_{27} \mathrm{H}_{25} \mathrm{NO}_{3}$ : C, 78.81; H, 6.12; N, 3.40. Found: C, 78.80; H, 6.14; N, 3.38. Second diastereomer: ${ }^{1} \mathrm{H}$ NMR $\left(300 \mathrm{MHz}, \mathrm{CDCl}_{3}\right) \delta_{\mathrm{H}} 0.58\left(3 \mathrm{H}, \mathrm{t}, J=7.2 \mathrm{~Hz}, \mathrm{CH}_{2} \mathrm{CH}_{3}\right), 1.46(1 \mathrm{H}, \mathrm{ddq}, J=6.6,13.8,7.2$ $\left.\mathrm{Hz}, \mathrm{CH}_{2} \mathrm{CH}_{3}\right), 1.59\left(1 \mathrm{H}, \mathrm{ddq}, J=6.9,13.8,7.2 \mathrm{~Hz}, \mathrm{CH}_{2} \mathrm{CH}_{3}\right), 3.92\left(1 \mathrm{H}, \mathrm{d}, J=15.0 \mathrm{~Hz}, \mathrm{CH}_{2} \mathrm{Ph}\right)$, $4.89\left(1 \mathrm{H}, \mathrm{d}, J=15.0 \mathrm{~Hz}, \mathrm{CH}_{2} \mathrm{Ph}\right), 5.18(1 \mathrm{H}, \mathrm{bs}, C H \mathrm{Ph}), 5.26(1 \mathrm{H}$, ddd, J=6.6, 6.9, $9.0 \mathrm{~Hz}$, CHOCOPh), $6.11(1 \mathrm{H}, \mathrm{d}, J=9.0 \mathrm{~Hz}, \mathrm{CH}=\mathrm{C}), 7.32-7.48(13 \mathrm{H}, \mathrm{m}, \mathrm{Ar}), 7.98$ (2H, d, J=8.1 Hz, Ar); ${ }^{13} \mathrm{C}$ NMR $\left(75 \mathrm{MHz}, \mathrm{CDCl}_{3}\right) \quad \delta_{\mathrm{C}} 8.8,26.9,44.2,62.4,71.7,122.6,127.5,127.7,128.3,128.5$, 128.7, 128.8, 128.9, 129.5, 129.6, 132.9, 133.0,136.3, 146.0, 165.5, 168.8; LC-ESI-MS rt 18.6 min., m/z $412(\mathrm{M}+1), 434(\mathrm{M}+\mathrm{Na}), 823(2 \mathrm{M}+1), 845(2 \mathrm{M}+\mathrm{Na})$; IR (film): 2970, 2928, 1710, 1654, 1456, $1268 \mathrm{~cm}^{-1}$; Calcd for $\mathrm{C}_{27} \mathrm{H}_{25} \mathrm{NO}_{3}$ : C, 78.81; H, 6.12; N, 3.40. Found: C, 78.81; H, $6.13 ; \mathrm{N}, 3.42$.

Ethyl 3-((E)-3-(2-benzoyloxybutylidene)-2-oxo-4-phenylazetidin-1-yl)propanoate (3c). ${ }^{1} \mathrm{H}$ NMR $\left(300 \mathrm{MHz}, \mathrm{CDCl}_{3}\right) \delta_{\mathrm{H}} 0.90\left(3 \mathrm{H}, \mathrm{t}, \mathrm{J}=7.6 \mathrm{~Hz}, \mathrm{CH}_{2} \mathrm{CH}_{3}\right), 1.20(3 \mathrm{H}, \mathrm{t}, \mathrm{J}=7.2 \mathrm{~Hz}$, $\left.\mathrm{COCH}_{2} \mathrm{CH}_{3}\right), 1.65\left(2 \mathrm{H}, \mathrm{m}, \mathrm{CH}_{2} \mathrm{CH}_{3}\right), 2.48(2 \mathrm{H}, \mathrm{m}), 3.27\left(1 \mathrm{H}, \mathrm{dt}, \mathrm{J}=14.2,7.0 \mathrm{~Hz}, \mathrm{CH}_{2} \mathrm{COO}\right), 3$. $68\left(1 \mathrm{H}, \mathrm{dt}, J=14.2,6.6 \mathrm{~Hz}, \mathrm{CH}_{2} \mathrm{COO}\right), 4.05\left(2 \mathrm{H}, \mathrm{q}, J=7.2 \mathrm{~Hz}, \mathrm{COCH}_{2} \mathrm{CH}_{3}\right), 4.97(1 \mathrm{H}, \mathrm{bs}$, CHPh), $5.30(1 \mathrm{H}$, pseudo quartet, $J=6.6 \mathrm{~Hz}, C H O C O P h), 6.32(1 \mathrm{H}, \mathrm{d}, J=6.6 \mathrm{~Hz}, C H=\mathrm{C}), 7.20$ $7.60(8 \mathrm{H}, \mathrm{m}, \mathrm{Ar}), 7.70-7.90(2 \mathrm{H}, \mathrm{m}, \mathrm{Ar}) ;{ }^{13} \mathrm{C} \mathrm{NMR}\left(75 \mathrm{MHz}, \mathrm{CDCl}_{3}\right) \delta_{\mathrm{C}} 9.4,14.0,27.2,33.0$, $36.3,60.7,63.7,72.3,124.2,127.9,128.1,128.4,128.7,129.6,130.0,133.5,135.6,143.1,165.2$, 171.0; LC-ESI-MS rt 16.7 min., m/z $422(\mathrm{M}+1), 865$ (2M+Na); IR (film): 3062, 3033, 2980, 2933, 2367,1754, 1725, $1377 \mathrm{~cm}^{-1}$; Calcd for $\mathrm{C}_{25} \mathrm{H}_{27} \mathrm{NO}_{5}$ : C, 71.24; H, 6.46; N, 3.32. Found: C, $71.22 ; \mathrm{H}, 6.48 ; \mathrm{N}, 3.30$.

(Z)-1-(1-Benzyl-2-oxo-4-phenylazetidin-3-ylidene)butan-2-yl 2-phenylacetate (4b). First diastereomer: ${ }^{1} \mathrm{H}$ NMR $\left(300 \mathrm{MHz}, \mathrm{CDCl}_{3}\right) \delta_{\mathrm{H}} 0.95\left(3 \mathrm{H}, \mathrm{t}, J=7.5 \mathrm{~Hz}, \mathrm{CH}_{2} \mathrm{CH}_{3}\right), 1.70-1.85(2 \mathrm{H}, \mathrm{m}$, $\left.\mathrm{CH}_{2} \mathrm{CH}_{3}\right), 3.71\left(2 \mathrm{H}, \mathrm{s}, \mathrm{COCH}_{2} \mathrm{Ph}\right), 3.87\left(1 \mathrm{H}, \mathrm{d}, \mathrm{J}=15.0 \mathrm{~Hz}, \mathrm{CH}_{2} \mathrm{Ph}\right), 4.78(1 \mathrm{H}, \mathrm{bs}, \mathrm{CHPh}) 4.90$ $\left(1 \mathrm{H}, \mathrm{d}, J=15.0 \mathrm{~Hz}, \mathrm{CH}_{2} \mathrm{Ph}\right), 5.38\left(1 \mathrm{H}, \mathrm{ddd}, J=7.5,4.2, J_{1,3}=1.0 \mathrm{~Hz}, C H O C O P h\right), 5.90(1 \mathrm{H}, \mathrm{d}$, $J=7.5 \mathrm{~Hz}, \mathrm{CH}=\mathrm{C}), 7.15-7.45(15 \mathrm{H}, \mathrm{m}, \mathrm{Ar}) ;{ }^{13} \mathrm{C} \mathrm{NMR}\left(75 \mathrm{MHz}, \mathrm{CDCl}_{3}\right) \delta_{\mathrm{C}} 9.1,26.7,41.4,44.1$, $61.2,72.3,125.9,126.7,126.9,127.2,127.5,128.2,128.5,128.7,129.0,129.1,134.1,135.2$, 144.6, 162.4, 170.6; LC-ESI-MS rt $18.3 \mathrm{~min} ., \mathrm{m} / \mathrm{z} 427$ (M+1), 449 (M+Na); IR (film): 3063, 3031, 2967, 2850, 1748, 1661, $1381 \mathrm{~cm}^{-1}$; Calcd for $\mathrm{C}_{28} \mathrm{H}_{27} \mathrm{NO}_{3}$ : C, 79.03; H, 6.40; N, 3.29. Found: C, 79.00; H, 6.38; N, 3.29. Second diastereomer: ${ }^{1} \mathrm{H}$ NMR $\left(300 \mathrm{MHz}, \mathrm{CDCl}_{3}\right) \delta_{\mathrm{H}} 0.75$ $\left(3 \mathrm{H}, \mathrm{t}, J=7.5 \mathrm{~Hz}, \mathrm{CH}_{2} \mathrm{CH}_{3}\right), 1.65-1.82\left(2 \mathrm{H}, \mathrm{m}, \mathrm{CH}_{2} \mathrm{CH}_{3}\right), 3.42\left(2 \mathrm{H}, \mathrm{s}, \mathrm{COCH}_{2} \mathrm{Ph}\right), 3.91(1 \mathrm{H}, \mathrm{d}$, 
$\left.J=15.0 \mathrm{~Hz}, \mathrm{NCH}_{2} \mathrm{Ph}\right), 4.43(1 \mathrm{H}, \mathrm{s}, \mathrm{CHPh}), 4.91\left(1 \mathrm{H}, \mathrm{d}, J=15.0 \mathrm{~Hz}, \mathrm{CH}_{2} \mathrm{Ph}\right), 5.00(1 \mathrm{H}, \mathrm{m}$, CHOCOPh), $5.87(1 \mathrm{H}, \mathrm{d}, \mathrm{J}=8.7 \mathrm{~Hz}, \mathrm{CH}=\mathrm{C}), 7.18-7.40(15 \mathrm{H}, \mathrm{m}, \mathrm{Ar}) ;{ }^{13} \mathrm{C} \mathrm{NMR}\left(75 \mathrm{MHz}, \mathrm{CDCl}_{3}\right)$ $\delta_{\mathrm{C}} 10.8,27.3,41.3,44.0,60.5,72.4,125.9,126.7,126.9,127.5,128.2,128.4,128.5,128.7$, 129.0, 129.1, 134.0, 136.1, 144.3, 162.4, 170.5; LC-ESI-MS rt $18.5 \mathrm{~min} ., \mathrm{m} / \mathrm{z} 427$ (M+1), 449 $(\mathrm{M}+\mathrm{Na}), 853(2 \mathrm{M}+1)$; IR (film): 3087, 3032, 2925, 1746, 1661, 1495; Calcd for $\mathrm{C}_{28} \mathrm{H}_{27} \mathrm{NO}_{3}$ : C, 79.03; H, 6.40; N, 3.29. Found: C, 79.01; H, 6.43; N, 3.28.

(E)-1-(1-Benzyl-2-oxo-4-phenylazetidin-3-ylidene)butan-2-yl 2-phenylacetate (4b). First diastereomer: ${ }^{1} \mathrm{H}$ NMR $\left(300 \mathrm{MHz}, \mathrm{CDCl}_{3}\right) \delta_{\mathrm{H}} 0.76\left(3 \mathrm{H}, \mathrm{t}, J=7.5 \mathrm{~Hz}, \mathrm{CH}_{2} \mathrm{CH}_{3}\right), 1.26-1.56(2 \mathrm{H}, \mathrm{m}$, $\left.\mathrm{CH}_{2} \mathrm{CH}_{3}\right), 3.38\left(2 \mathrm{H}, \mathrm{s}, \mathrm{COCH}_{2} \mathrm{Ph}\right), 3.80\left(1 \mathrm{H}, \mathrm{d}, J=15.0 \mathrm{~Hz}, \mathrm{CH}_{2} \mathrm{Ph}\right), 4.84\left(1 \mathrm{H}, \mathrm{d}, J_{1,3}=1.5 \mathrm{~Hz}\right.$, CHPh) $4.87\left(1 \mathrm{H}, \mathrm{d}, J=15.0 \mathrm{~Hz}, \mathrm{CH}_{2} \mathrm{Ph}\right), 5.00(1 \mathrm{H}, \mathrm{m}, C H O C O P h), 6.22(1 \mathrm{H}, \mathrm{dd}, J=6.3 \mathrm{~Hz}$, $\left.J_{1,3}=1.5 \mathrm{~Hz}, \mathrm{CH}=\mathrm{C}\right), 7.15-7.45(15 \mathrm{H}, \mathrm{m}, \mathrm{Ar}) ;{ }^{13} \mathrm{C} \mathrm{NMR}\left(75 \mathrm{MHz}, \mathrm{CDCl}_{3}\right) \delta_{\mathrm{C}} 9.0,27.0,41.0$, $44.1,62.2,71.9,123.8,127.0,127.4,127.6,127.8,128.4,128.7,128.8,128.9,129.0,135.2$, 136.3, 145.7, 163.5, 170.0; LC-ESI-MS rt 16.0 min., m/z 427 (M+1), 449 (M+Na) 853 (2M+1); IR (film): 3064, 3031, 2926, 1751, 1456, 1383; Calcd for $\mathrm{C}_{28} \mathrm{H}_{27} \mathrm{NO}_{3}$ : C, 79.03; H, 6.40; N, 3.29. Found: C, 79.04; H, 6.42; N, 3.26. Second diastereomer: ${ }^{1} \mathrm{H}$ NMR $\left(300 \mathrm{MHz}, \mathrm{CDCl}_{3}\right) \delta_{\mathrm{H}} 0.45$ $\left(3 \mathrm{H}, \mathrm{t}, J=7.5 \mathrm{~Hz}, \mathrm{CH}_{2} \mathrm{CH}_{3}\right), 1.28-1.52\left(2 \mathrm{H}, \mathrm{m}, \mathrm{CH}_{2} \mathrm{CH}_{3}\right), 3.42\left(1 \mathrm{H}, \mathrm{d}, J=15.0 \mathrm{~Hz}, \mathrm{COCH}_{2} \mathrm{Ph}\right)$, $3.50\left(1 \mathrm{H}, \mathrm{d}, J=15.0 \mathrm{~Hz}, \mathrm{COCH}_{2} \mathrm{Ph}\right), 3.90\left(1 \mathrm{H}, \mathrm{d}, J=15.0 \mathrm{~Hz}, \mathrm{NCH}_{2} \mathrm{Ph}\right), 4.87(1 \mathrm{H}, \mathrm{d}, J=15.0 \mathrm{~Hz}$, $\left.\mathrm{CH}_{2} \mathrm{Ph}\right), 5.01(1 \mathrm{H}, \mathrm{m}, \mathrm{CHOCOPh}), 5.09\left(1 \mathrm{H}, \mathrm{d}, J_{1,3}=1.5 \mathrm{~Hz}, C H \mathrm{Ph}\right), 6.00(1 \mathrm{H}, \mathrm{dd}, J=8.7 \mathrm{~Hz}$, $\left.J_{1,3}=1.5 \mathrm{~Hz}, C H=\mathrm{C}\right), 7.15-7.45(15 \mathrm{H}, \mathrm{m}, \mathrm{Ar}) ;{ }^{13} \mathrm{C} \mathrm{NMR}\left(75 \mathrm{MHz}, \mathrm{CDCl}_{3}\right) \delta_{\mathrm{C}} 8.5,26.6,41.0$, $44.2,62.3,71.6,122.4,127.0,127.5,127.7,127.8,128.4,128.6,128.8,128.9,129.1,135.0$, 135.4, 145.7, 163.4, 170.7; LC-ESI-MS rt $17.3 \mathrm{~min} ., \mathrm{m} / \mathrm{z} 427$ (M+1), 449 (M+Na); IR (film): 3063, 2924, 2854, 1712, 1655, 1457, 1377; Calcd for $\mathrm{C}_{28} \mathrm{H}_{27} \mathrm{NO}_{3}$ : C, 79.03; H, 6.40; N, 3.29. Found: C, 79.02; H, 6.40; N, 3.27.

(Z)-1-(1-Benzyl-2-(4-nitrophenyl)-4-oxoazetidin-3-ylidene)butan-2-yl 2-phenylacetate (4d). First diastereomer: ${ }^{1} \mathrm{H}$ NMR $\left(300 \mathrm{MHz}, \mathrm{CDCl}_{3}\right) \delta_{\mathrm{H}} 0.94\left(3 \mathrm{H}, \mathrm{t}, J=7.5 \mathrm{~Hz}, \mathrm{CH}_{2} \mathrm{CH}_{3}\right), 1.60-1.80$ $\left(2 \mathrm{H}, \mathrm{m}, \mathrm{CH}_{2} \mathrm{CH}_{3}\right), 3.65\left(2 \mathrm{H}, \mathrm{s}, \mathrm{COOCH}_{2} \mathrm{Ph}\right), 3.98\left(1 \mathrm{H}, \mathrm{d}, \mathrm{J}=15.0 \mathrm{~Hz}, \mathrm{CH}_{2} \mathrm{Ph}\right), 4.82(1 \mathrm{H}, \mathrm{bs}$, CHPh), $4.85\left(1 \mathrm{H}, \mathrm{d}, J=15.0 \mathrm{~Hz}, \mathrm{CH}_{2} \mathrm{Ph}\right), 5.32(1 \mathrm{H}, \mathrm{d}, J=7.8 \mathrm{~Hz}, C H=\mathrm{C}), 5.81(1 \mathrm{H}$, broad dt=q, $J=7.2 \mathrm{~Hz}, C H O C O P h), 7.10-7.20(2 \mathrm{H}, \mathrm{m}, \mathrm{Ar}), 7.22-7.40(8 \mathrm{H}, \mathrm{m}, \mathrm{Ar}), 7.40(2 \mathrm{H}, \mathrm{d}, J=8.7 \mathrm{~Hz}$, Ar), $8.15(2 \mathrm{H}, \mathrm{t}, J=8.7 \mathrm{~Hz}, \mathrm{Ar}) ;{ }^{13} \mathrm{C} \mathrm{NMR}\left(75 \mathrm{MHz}, \mathrm{CDCl}_{3}\right) \delta_{\mathrm{C}} 9.1,27.3,41.4,44.8,60.5,72.4$, $124.0,127.0,127.9,128.0,128.4,128.5,128.6,128.8,129.1,133.9,134.6,137.4,144.0,148.0$, 162.1, 170.8; LC-ESI-MS rt $16.5 \mathrm{~min} ., \mathrm{m} / \mathrm{z} 471$ (M+1), 493 (M+Na); IR (film): 3010, 2925, 1751, 1653, 1521, 1457, 1349, $1073 \mathrm{~cm}^{-1}$; Calcd for $\mathrm{C}_{28} \mathrm{H}_{26} \mathrm{~N}_{2} \mathrm{O}_{5}$ : C, 71.47; H, 5.57; N, 5.95. Found: C, 71.49; H, 5.55; N, 5.92. Second diastereomer: ${ }^{1} \mathrm{H}$ NMR $\left(300 \mathrm{MHz}, \mathrm{CDCl}_{3}\right) \delta_{\mathrm{H}} 0.89$ $\left(3 \mathrm{H}, \mathrm{t}, \mathrm{J}=7.5 \mathrm{~Hz}, \mathrm{CH}_{2} \mathrm{CH}_{3}\right), 1.60-1.80\left(2 \mathrm{H}, \mathrm{m}, \mathrm{CH}_{2} \mathrm{CH}_{3}\right), 3.70\left(2 \mathrm{H}, \mathrm{s}, \mathrm{COOCH}_{2} \mathrm{Ph}\right), 4.02(1 \mathrm{H}, \mathrm{d}$, $\left.J=15.0 \mathrm{~Hz}, \mathrm{CH}_{2} \mathrm{Ph}\right), 4.84(1 \mathrm{H}, \mathrm{s}, \mathrm{CHPh}), 4.87\left(1 \mathrm{H}, \mathrm{d}, J=15.0 \mathrm{~Hz}, \mathrm{CH}_{2} \mathrm{Ph}\right), 5.37(1 \mathrm{H}, \mathrm{d}, J=7.2 \mathrm{~Hz}$, $\mathrm{CH}=\mathrm{C}), 5.77(1 \mathrm{H}, \mathrm{q}, J=7.2 \mathrm{~Hz}, \mathrm{CHOCOPh}), 7.10-7.20$ (2H, m, Ar), 7.22-7.45 (10H, m, Ar), 8.21 $(2 \mathrm{H}, \mathrm{d}, J=8.4 \mathrm{~Hz}, \mathrm{Ar}) ;{ }^{13} \mathrm{C} \mathrm{NMR}\left(75 \mathrm{MHz}, \mathrm{CDCl}_{3}\right) \delta_{\mathrm{C}} 9.2,27.5,41.4,45.0,60.6,72.4,124.1$, 127.0, 128.0, 128.4, 128.5, 128.6, 128.8, 129.1, 129.3, 134.0, 134.5, 137.3, 143.5, 148.1, 162.1, 170.8; LC-ESI-MS rt 16.6 min., m/z $471(\mathrm{M}+1), 493$ (M+Na); IR (film): 2995, 2925, 1751, 
1637, 1522, 1455, 1349, 1259, 1073, $1073 \mathrm{~cm}^{-1}$; Calcd for $\mathrm{C}_{28} \mathrm{H}_{26} \mathrm{~N}_{2} \mathrm{O}_{5}: \mathrm{C}, 71.47 ; \mathrm{H}, 5.57 ; \mathrm{N}$, 5.95. Found: C, 71.45; H, 5.56; N, 5.95.

(E)-1-(1-Benzyl-2-(4-nitrophenyl)-4-oxoazetidin-3-ylidene)butan-2-yl 2-phenylacetate (4d). ${ }^{1} \mathrm{H}$ NMR $\left(300 \mathrm{MHz}, \mathrm{CDCl}_{3}\right) \delta_{\mathrm{H}} 0.44\left(3 \mathrm{H}, \mathrm{t}, J=7.5 \mathrm{~Hz}, \mathrm{CH}_{2} \mathrm{CH}_{3}\right), 1.33-1.51\left(2 \mathrm{H}, \mathrm{m}, \mathrm{CH}_{2} \mathrm{CH}_{3}\right)$, $3.52\left(1 \mathrm{H}, \mathrm{d}, J=15.6 \mathrm{~Hz}, \mathrm{COOCH}_{2} \mathrm{Ph}\right), 3.58\left(1 \mathrm{H}, \mathrm{d}, J=15.6 \mathrm{~Hz}, \mathrm{COOCH}_{2} \mathrm{Ph}\right), 4.05(1 \mathrm{H}, \mathrm{d}, J=15.0$ $\left.\mathrm{Hz}, \mathrm{CH}_{2} \mathrm{Ph}\right), 4.80\left(1 \mathrm{H}, \mathrm{d}, J=15.0 \mathrm{~Hz}, \mathrm{CH}_{2} \mathrm{Ph}\right), 4.84(1 \mathrm{H}, \mathrm{dt}, J=6.9,9.3 \mathrm{~Hz}, \mathrm{CHOCOPh}), 5.16$ $(1 \mathrm{H}, \mathrm{s}, C H \mathrm{Ph}), 5.01(1 \mathrm{H}, \mathrm{d}, J=9.3 \mathrm{~Hz}, C H=\mathrm{C}), 7.10-7.15$ (2H, m, Ar), 7.20-7.40 (8H, m, Ar), $7.46(2 \mathrm{H}, \mathrm{d}, J=8.4 \mathrm{~Hz}, \mathrm{Ar}), 8.19(2 \mathrm{H}, \mathrm{d}, J=8.4 \mathrm{~Hz}, \mathrm{Ar}) ;{ }^{13} \mathrm{C} \mathrm{NMR}\left(75 \mathrm{MHz}, \mathrm{CDCl}_{3}\right) \delta_{\mathrm{C}} 8.7,26.9$, 41.2, 45.0, 61.5, 71.3, 123.3, 124.0, 127.2, 128.1, 128.4, 128.5, 128.6, 128.8, 129.1, 133.4, 134.3, 144.2, 145.7, 148.0, 163.1, 170.9; LC-ESI-MS rt $15.7 \mathrm{~min} ., \mathrm{m} / \mathrm{z} 471(\mathrm{M}+1), 493$ (M+Na); IR (film): 2995, 2926, 1756, 1637, 1522, 1456, 1349, 1258, 1157, $1074 \mathrm{~cm}^{-1}$; Calcd for $\mathrm{C}_{28} \mathrm{H}_{26} \mathrm{~N}_{2} \mathrm{O}_{5}$ : C, 71.47; H, 5.57; N, 5.95. Found: C, 71.48; H, 5.60; N, 5.96.

\section{General procedure for the hydrolysis of ester $\mathbf{3}$ or $\mathbf{4}$ to hydroxy-derivatives $\mathbf{5}$}

Compound 3 or 4 ( $1 \mathrm{mmol}$ ) was dissolved in a $0.62 \mathrm{M}$ solution of $\mathrm{NaOH}$ in methanol (3 ml) and stirred at room temperature for $72 \mathrm{~h}$. The reaction was then diluted with water $(10 \mathrm{ml})$ and methanol removed under reduced pressure. The residue was then extracted twice with $\mathrm{CH}_{2} \mathrm{Cl}_{2}$ $(10 \mathrm{~mL})$. The organic layers were dried over $\mathrm{Na}_{2} \mathrm{SO}_{4}$ and solvent was removed under reduced pressure. Hydroxyderivative $\mathbf{5}$ was isolated by flash chromatography on silica gel (cyclohexane/ethyl acetate 8/2).

(Z)-1-Benzyl-3-(2-hydroxyethylidene)-4-phenylazetidin-2-one (5a). ${ }^{1} \mathrm{H}$ NMR (300MHz, $\left.\mathrm{CDCl}_{3}\right) \delta_{\mathrm{H}} 3.92\left(1 \mathrm{H}, \mathrm{d}, J=15.0 \mathrm{~Hz}, \mathrm{CH}_{2} \mathrm{Ph}\right), 4.42\left(2 \mathrm{H}, \mathrm{d}, J=4.0 \mathrm{~Hz}, \mathrm{CH}_{2} \mathrm{OH}\right), 4.81(1 \mathrm{H}, \mathrm{s}, \mathrm{CHPh})$, $4.89\left(1 \mathrm{H}, \mathrm{d}, J=15.0 \mathrm{~Hz}, \mathrm{CH}_{2} \mathrm{Ph}\right), 5.75(1 \mathrm{H}, \mathrm{t}, J=4.0 \mathrm{~Hz}, \mathrm{CH}=\mathrm{C}), 7.00-7.40(10 \mathrm{H}, \mathrm{m}, \mathrm{Ar}) ;{ }^{13} \mathrm{C}$ NMR $\left(75 \mathrm{MHz}, \mathrm{CDCl}_{3}\right) \quad \delta_{\mathrm{C}} 44.7,61.3,61.4,127.4,127.9,128.5,128.8,128.9,129.0,130.5$, 134.9, 135.8, 140.8, 164.4; LC-ESI-MS rt 10.2 min., m/z 280 (M+1), 302 (M+Na); IR (film) 3417, 3039, 2952, 2906, 1606, 1454, 1375, 1250, 1103, $1010 \mathrm{~cm}^{-1}$; Calcd for $\mathrm{C}_{18} \mathrm{H}_{17} \mathrm{NO}_{2}: \mathrm{C}$, 77.40; H, 6.13; N, 5.01. Found: C, 77.42; H, 6.12; N, 5.00.

(E)-1-Benzyl-3-(2-hydroxyethylidene)-4-phenylazetidin-2-one (5a). ${ }^{1} \mathrm{H}$ NMR (300MHz, $\left.\mathrm{CDCl}_{3}\right) \delta_{\mathrm{H}} 2.40(1 \mathrm{H}, \mathrm{bs}, \mathrm{OH}), 3.80\left(1 \mathrm{H}, \mathrm{d}, J=15.0 \mathrm{~Hz}, \mathrm{CH}_{2} \mathrm{Ph}\right), 4.04\left(2 \mathrm{H}, \mathrm{d}, J=5.1 \mathrm{~Hz}, \mathrm{CH}_{2} \mathrm{OH}\right)$, $4.87\left(1 \mathrm{H}, \mathrm{d}, J=15.0 \mathrm{~Hz}, \mathrm{CH}_{2} \mathrm{Ph}\right), 4.97(1 \mathrm{H}, \mathrm{s}, \mathrm{CHPh}), 6.27(1 \mathrm{H}, \mathrm{t}, J=5.1 \mathrm{~Hz}, C H=\mathrm{C}), 7.15(2 \mathrm{H}, \mathrm{d}$, $J=6.6 \mathrm{~Hz}, \mathrm{Ar}), 7.25-7.40(8 \mathrm{H}, \mathrm{m}, \mathrm{Ar}) ;{ }^{13} \mathrm{C} \mathrm{NMR}\left(75 \mathrm{MHz}, \mathrm{CDCl}_{3}\right) \delta_{\mathrm{C}} 44.1,59.5,62.1,124.7$, 127.5, 127.7, 128.3, 128.7, 128.8, 128.9, 135.2, 136.6, 142.6, 164.0; LC-ESI-MS rt 9.6 min., m/z $280(\mathrm{M}+1), 302(\mathrm{M}+\mathrm{Na})$; IR (film) 2966, 2846, 1646, 1394, 1262, 1096, $1010 \mathrm{~cm}^{-1}$; Calcd for $\mathrm{C}_{18} \mathrm{H}_{17} \mathrm{NO}_{2}$ : C, 77.40; H, 6.13; N, 5.01. Found: C, 77.37; H, 6.16; N, 5.04.

(Z)-1-Benzyl-3-(2-hydroxybutylidene)-4-phenylazetidin-2-one (5b). First diastereomer: ${ }^{1} \mathrm{H}$ NMR $\left(300 \mathrm{MHz}, \mathrm{CDCl}_{3}\right) \delta_{\mathrm{H}} 0.99\left(3 \mathrm{H}, \mathrm{t}, J=7.5 \mathrm{~Hz}, \mathrm{CH}_{2} \mathrm{CH}_{3}\right), 1.60-1.68\left(2 \mathrm{H}, \mathrm{m}, \mathrm{CH}_{2} \mathrm{CH}_{3}\right), 3.92$ $\left(1 \mathrm{H}, \mathrm{d}, \mathrm{J}=15.0 \mathrm{~Hz}, \mathrm{CH}_{2} \mathrm{Ph}\right), 4.51(1 \mathrm{H}, \mathrm{m}, \mathrm{CHOH}), 4.81(1 \mathrm{H}, \mathrm{bs}, \mathrm{CHPh}), 4.90(1 \mathrm{H}, \mathrm{d}, \mathrm{J}=15.0 \mathrm{~Hz}$, $\left.\mathrm{CH}_{2} \mathrm{Ph}\right), 5.72\left(1 \mathrm{H}, \mathrm{d}, J=4.8 \mathrm{~Hz}, J_{1,3}=1.0 \mathrm{~Hz}, \mathrm{CH}=\mathrm{C}\right), 7.17-7.41(10 \mathrm{H}, \mathrm{m}, \mathrm{Ar}) ;{ }^{13} \mathrm{C} \mathrm{NMR}(75 \mathrm{MHz}$, $\left.\mathrm{CDCl}_{3}\right) \delta_{\mathrm{C}} 9.5,30.3,44.6,61.4,71.3,127.4,127.8,128.3,128.4,128.5,128.8,128.9,134.0$, 135.9, 140.9, 162.1; LC-ESI-MS rt 12.3 min., m/z $308(\mathrm{M}+1), 330(\mathrm{M}+\mathrm{Na}), 637$ (2M+Na); IR 
(film): 3420, 3063, 2924, 1733, 1455, 1388, $1109 \mathrm{~cm}^{-1}$; Calcd for $\mathrm{C}_{18} \mathrm{H}_{17} \mathrm{NO}_{2}$ : C, 78.15; $\mathrm{H}, 6.89$; $\mathrm{N}$, 4.56. Found: C, 78.11; H, 6.90; N, 4.56. Second diastereomer: ${ }^{1} \mathrm{H}$ NMR $\left(300 \mathrm{MHz}, \mathrm{CDCl}_{3}\right) \delta_{\mathrm{H}}$ $1.00\left(3 \mathrm{H}, \mathrm{t}, J=7.5 \mathrm{~Hz}, \mathrm{CH}_{2} \mathrm{CH}_{3}\right), 1.63-1.71\left(2 \mathrm{H}, \mathrm{m}, \mathrm{CH}_{2} \mathrm{CH}_{3}\right), 3.95\left(1 \mathrm{H}, \mathrm{d}, J=15.0 \mathrm{~Hz}, \mathrm{CH}_{2} \mathrm{Ph}\right)$, $4.64(1 \mathrm{H}, \mathrm{m}, \mathrm{CHOH}), 4.83(1 \mathrm{H}, \mathrm{bs}, \mathrm{CHPh}), 4.90\left(1 \mathrm{H}, \mathrm{d}, J=15.0 \mathrm{~Hz}, \mathrm{CH}_{2} \mathrm{Ph}\right), 5.90(1 \mathrm{H}, \mathrm{d}, J=4.5$ $\left.\mathrm{Hz}, J_{1,3}=1.0 \mathrm{~Hz}, \mathrm{CH}=\mathrm{C}\right), 7.19-7.40(10 \mathrm{H}, \mathrm{m}, \mathrm{Ar}) ;{ }^{13} \mathrm{C} \mathrm{NMR}\left(75 \mathrm{MHz}, \mathrm{CDCl}_{3}\right) \delta_{\mathrm{C}} 9.2,30.3,44.4$, $61.3,71.4,127.3,127.8,128.2,128.4,128.5,128.7,128.9,133.8,136.0,140.7,162.7$; LC-ESIMS rt 12.1 min., m/z 308 (M+1), 330 (M+Na), 637 (2M+Na); IR (film): 3421, 3064, 3031, 2963, 1731, 1455, 1353, $1069 \mathrm{~cm}^{-1}$; Calcd for $\mathrm{C}_{20} \mathrm{H}_{21} \mathrm{NO}_{2}$ : C, 78.15; H, 6.89; N, 4.56. Found: $\mathrm{C}, 78.16$; $\mathrm{H}, 6.85 ; \mathrm{N}, 4.53$.

(E)-1-Benzyl-3-(2-hydroxybutylidene)-4-phenylazetidin-2-one (5b). First diastereomer: ${ }^{1} \mathrm{H}$ NMR $\left(300 \mathrm{MHz}, \mathrm{CDCl}_{3}\right) \delta_{\mathrm{H}} 0.80\left(3 \mathrm{H}, \mathrm{t}, J=7.5 \mathrm{~Hz}, \mathrm{CH}_{2} \mathrm{CH}_{3}\right), 1.46\left(2 \mathrm{H}, \mathrm{m}, \mathrm{CH}_{2} \mathrm{CH}_{3}\right), 3.84(1 \mathrm{H}, \mathrm{d}$, $\left.J=15.0 \mathrm{~Hz}, \mathrm{CH}_{2} \mathrm{Ph}\right), 4.00(1 \mathrm{H}, \mathrm{dt}, J=6.3,6.6 \mathrm{~Hz}, \mathrm{CHOH}), 4.89\left(1 \mathrm{H}, \mathrm{d}, J=15.0 \mathrm{~Hz}, \mathrm{CH}_{2} \mathrm{Ph}\right), 4.95$ $(1 \mathrm{H}, \mathrm{s}, \mathrm{CHPh}), 6.18(1 \mathrm{H}, \mathrm{d}, J=6.6 \mathrm{~Hz}, \mathrm{CH}=\mathrm{C}), 7.15-7.40(10 \mathrm{H}, \mathrm{m}, \mathrm{Ar}) ;{ }^{13} \mathrm{C}$ NMR $(75 \mathrm{MHz}$, $\left.\mathrm{CDCl}_{3}\right) \delta_{\mathrm{C}} 9.2,29.7,44.2,62.2,70.6,127.6,127.7,127.8,128.4,128.9,129.0,135.3,136.5$, 142.9, 164.1; LC-ESI-MS rt $11.1 \mathrm{~min} ., \mathrm{m} / \mathrm{z} 308$ (M+1), 330 (M+Na); IR (film): 3432, 2959, 2924, 2853, 1736, 1641, 1454, $1111 \mathrm{~cm}^{-1}$; Calcd for $\mathrm{C}_{20} \mathrm{H}_{21} \mathrm{NO}_{2}$ : C, 78.15; H, 6.89; N, 4.56. Found: C, 78.18; H, 6.94; N, 4.57. Second diastereomer: ${ }^{1} \mathrm{H}$ NMR $\left(300 \mathrm{MHz}, \mathrm{CDCl}_{3}\right) \delta_{\mathrm{H}} 0.64$ $\left(3 \mathrm{H}, \mathrm{t}, J=7.4 \mathrm{~Hz}, \mathrm{CH}_{2} \mathrm{CH}_{3}\right), 1.38\left(2 \mathrm{H}, \mathrm{m}, \mathrm{CH}_{2} \mathrm{CH}_{3}\right), 3.72\left(1 \mathrm{H}, \mathrm{d}, J=15.0 \mathrm{~Hz}, \mathrm{CH}_{2} \mathrm{Ph}\right), 3.97(1 \mathrm{H}$, pseudo quartet, $J=6.6 \mathrm{~Hz}, \mathrm{CHOH}), 4.90\left(1 \mathrm{H}, \mathrm{d}, J=15.0 \mathrm{~Hz}, \mathrm{CH}_{2} \mathrm{Ph}\right), 4.99(1 \mathrm{H}, \mathrm{s}, \mathrm{CHPh}), 6.12$ $(1 \mathrm{H}, \mathrm{d}, J=6.6 \mathrm{~Hz}, \mathrm{CH}=\mathrm{C}), 7.17-7.38(10 \mathrm{H}, \mathrm{m}, \mathrm{Ar}) ;{ }^{13} \mathrm{C} \mathrm{NMR}\left(75 \mathrm{MHz}, \mathrm{CDCl}_{3}\right) \delta_{\mathrm{C}} 9.1,29.5$, 44.1, 62.2, 70.5, 127.5, 127.7, 127.8, 128.4, 128.6, 128.7, 128.8, 128.9, 135.3, 136.7, 142.9, 164.1; LC-ESI-MS rt 11.0 min., m/z 308 (M+1), $330(\mathrm{M}+\mathrm{Na}), 637$ (2M+Na); IR (film): 3430, 2958, 2953, 2860, 1661, 1453, $1110 \mathrm{~cm}^{-1}$; Calcd for $\mathrm{C}_{20} \mathrm{H}_{21} \mathrm{NO}_{2}: \mathrm{C}, 78.15 ; \mathrm{H}, 6.89 ; \mathrm{N}, 4.56$. Found: C, 78.14; H, 6.88; N, 4.54.

3-((E)-3-(2-Hydroxybutylidene)-2-oxo-4-phenylazetidin-1-yl)propanoic acid (5c). ${ }^{1} \mathrm{H}$ NMR $\left(300 \mathrm{MHz}, \mathrm{CDCl}_{3}\right) \delta_{\mathrm{H}} 0.80\left(3 \mathrm{H}, \mathrm{t}, J=7.2 \mathrm{~Hz}, \mathrm{CH}_{2} \mathrm{CH}_{3}\right), 1.46\left(2 \mathrm{H}, \mathrm{m}, \mathrm{CH}_{2} \mathrm{CH}_{3}\right), 2.62(2 \mathrm{H}, \mathrm{m}$, $\left.\mathrm{CH}_{2} \mathrm{CO}\right), 3.32\left(1 \mathrm{H}, \mathrm{dt}, J=6.6,14.6 \mathrm{~Hz}, \mathrm{NCH}_{2}\right), 3.75\left(1 \mathrm{H}, \mathrm{dt}, J=7.8,14.6 \mathrm{~Hz}, \mathrm{NCH}_{2}\right), 4.05(1 \mathrm{H}$, pseudo quartet, $J=6.2 \mathrm{~Hz}, \mathrm{CHOH}), 5.17(1 \mathrm{H}, \mathrm{bs}, \mathrm{CHPh}), 6.16(1 \mathrm{H}, \mathrm{d}, J=6.2 \mathrm{~Hz}, \mathrm{CH}=\mathrm{C}), 7.30-$ $7.70(5 \mathrm{H}, \mathrm{m}, \mathrm{Ar}) ;{ }^{13} \mathrm{C} \mathrm{NMR}\left(75 \mathrm{MHz}, \mathrm{CDCl}_{3}\right) \delta_{\mathrm{C}} 9.0,29.8,36.1,44.7,60.7,69.6,125.4,128.0$, 128.4, 128.8, 129.3, 138.1, 142.0, 164.3, 178.1; Calcd for $\mathrm{C}_{18} \mathrm{H}_{23} \mathrm{NO}_{4}: \mathrm{C}, 68.12 ; \mathrm{H}, 7.30 ; \mathrm{N}, 4.41$. Found: C, 68.14; H, 7.31; N, 4.40 .

(Z)-1-Benzyl-3-(2-hydroxybutylidene)-4-(4-nitrophenyl)azetidin-2-one $\mathbf{5 d}$. First diastereomer: ${ }^{1} \mathrm{H}$ NMR $\left(300 \mathrm{MHz}, \mathrm{CDCl}_{3}\right) \delta_{\mathrm{H}} 0.97\left(3 \mathrm{H}, \mathrm{t}, \mathrm{J}=7.2 \mathrm{~Hz}, \mathrm{CH}_{2} \mathrm{CH}_{3}\right), 1.54-1.67$ $\left(2 \mathrm{H}, \mathrm{m}, \mathrm{CH}_{2} \mathrm{CH}_{3}\right), 4.05\left(1 \mathrm{H}, \mathrm{d}, J=15.0 \mathrm{~Hz}, \mathrm{CH}_{2} \mathrm{Ph}\right), 4.53(1 \mathrm{H}, \mathrm{dt}, J=4.5,5.7 \mathrm{~Hz}, \mathrm{CHOH}), 4.89$ $(1 \mathrm{H}, \mathrm{bs}, C H \mathrm{Ph}), 4.90\left(1 \mathrm{H}, \mathrm{d}, J=15.0 \mathrm{~Hz}, \mathrm{CH}_{2} \mathrm{Ph}\right), 5.72\left(1 \mathrm{H}, \mathrm{dd}, J=4.5 \mathrm{~Hz}, J_{1,3}=1.5 \mathrm{~Hz}, C H=\mathrm{C}\right)$, 7.05-7.12 (2H, m, Ar), 7.14-7.40 (3H, m, Ar), $7.42(2 \mathrm{H}, \mathrm{d}, J=8.7 \mathrm{~Hz}, \mathrm{Ar}), 8.23(2 \mathrm{H}, \mathrm{d}, J=8.7 \mathrm{~Hz}$, $\mathrm{Ar}) ;{ }^{13} \mathrm{C} \mathrm{NMR}\left(75 \mathrm{MHz}, \mathrm{CDCl}_{3}\right) \delta_{\mathrm{C}} 9.3,30.2,45.3,60.5,71.3,124.2,126.9,128.1,128.2,128.6$, 128.9, 134.3, 135.1, 140.2, 143.7, 163.9; LC-ESI-MS rt 11.8 min., m/z $353(\mathrm{M}+1), 375(\mathrm{M}+\mathrm{Na})$; IR (film): 3419, 2964, 2926, 2854, 1734, 1654, 1522, 1496, 1349, 1221, $1105 \mathrm{~cm}^{-1} ; 1110 \mathrm{~cm}^{-1}$; Calcd for $\mathrm{C}_{20} \mathrm{H}_{20} \mathrm{~N}_{2} \mathrm{O}_{4}$ : C, 68.17; H, 5.72; N, 7.95. Found: C, 68.20; H, 5.74; N, 7.98. Second 
diastereomer ${ }^{1} \mathrm{H} \mathrm{NMR}\left(300 \mathrm{MHz}, \mathrm{CDCl}_{3}\right) \delta_{\mathrm{H}} 1.00\left(3 \mathrm{H}, \mathrm{t}, \mathrm{J}=7.2 \mathrm{~Hz}, \mathrm{CH}_{2} \mathrm{CH}_{3}\right), 1.58-1.70(2 \mathrm{H}, \mathrm{m}$, $\left.\mathrm{CH}_{2} \mathrm{CH}_{3}\right), 4.06\left(1 \mathrm{H}, \mathrm{d}, \mathrm{J}=15.0 \mathrm{~Hz}, \mathrm{CH}_{2} \mathrm{Ph}\right), 4.51(1 \mathrm{H}, \mathrm{dt}, \mathrm{J}=4.8,5.7 \mathrm{~Hz}, \mathrm{CHOH}), 4.88(1 \mathrm{H}, \mathrm{d}$, $\left.J=15.0 \mathrm{~Hz}, \mathrm{CH}_{2} \mathrm{Ph}\right), 4.91(1 \mathrm{H}, \mathrm{bs}, C H \mathrm{Ph}), 5.71\left(1 \mathrm{H}, \mathrm{dd}, J=4.8 \mathrm{~Hz}, J_{1,3}=1.5 \mathrm{~Hz}, \mathrm{CH}=\mathrm{C}\right), 7.05-$ $7.20(2 \mathrm{H}, \mathrm{m}, \mathrm{Ar}), 7.22-7.40(3 \mathrm{H}, \mathrm{m}, \mathrm{Ar}), 7.42(2 \mathrm{H}, \mathrm{d}, J=8.7 \mathrm{~Hz}, \mathrm{Ar}), 8.23$ (2H, d, J=8.7 Hz, Ar); ${ }^{13} \mathrm{C} \mathrm{NMR}\left(75 \mathrm{MHz}, \mathrm{CDCl}_{3}\right) \delta_{\mathrm{C}} 9.5,30.3,45.3,60.6,71.3,123.9,128.1,128.2,128.5,128.6$, 128.9, 134.3, 135.0, 140.3, 142.9, 162.6; LC-ESI-MS rt 11.7 min., m/z $353(\mathrm{M}+1), 375(\mathrm{M}+\mathrm{Na})$; IR (film): 3399, 2966, 2926, 2847, 1734, 1654, 1522, 1496, 1349, 1262, $1106 \mathrm{~cm}^{-1}$; Calcd for $\mathrm{C}_{20} \mathrm{H}_{20} \mathrm{~N}_{2} \mathrm{O}_{4}$ : C, 68.17; H, 5.72; N, 7.95. Found: C, 68.18; H, 5.70; N, 7.96.

(E)-1-Benzyl-3-(2-hydroxybutylidene)-4-(4-nitrophenyl)azetidin-2-one $\quad$ (5d). ${ }^{1} \mathrm{H} \quad \mathrm{NMR}$ $\left(300 \mathrm{MHz}, \mathrm{CDCl}_{3}\right) \delta_{\mathrm{H}} 0.76\left(3 \mathrm{H}, \mathrm{t}, J=7.5 \mathrm{~Hz}, \mathrm{CH}_{2} \mathrm{CH}_{3}\right), 1.43-1.54\left(2 \mathrm{H}, \mathrm{m}, \mathrm{CH}_{2} \mathrm{CH}_{3}\right), 3.92(1 \mathrm{H}, \mathrm{d}$, $\left.J=15.0 \mathrm{~Hz}, \mathrm{CH}_{2} \mathrm{Ph}\right), 4.01(1 \mathrm{H}, \mathrm{q}, J=5.4 \mathrm{~Hz}, \mathrm{CHOH}), 4.87\left(1 \mathrm{H}, \mathrm{d}, J=15.0 \mathrm{~Hz}, \mathrm{CH}_{2} \mathrm{Ph}\right), 5.10(1 \mathrm{H}$, s, CHPh), $6.22\left(1 \mathrm{H}, \mathrm{dd}, J=5.4 \mathrm{~Hz}, J_{1,3}=1.5 \mathrm{~Hz}, C H=\mathrm{C}\right), 7.12-7.15(2 \mathrm{H}, \mathrm{m}, \mathrm{Ar}), 7.30-7.42(3 \mathrm{H}$, m, Ar), $7.43(2 \mathrm{H}, \mathrm{d}, J=8.4 \mathrm{~Hz}, \mathrm{Ar}), 8.21(2 \mathrm{H}, \mathrm{d}, J=8.4 \mathrm{~Hz}, \mathrm{Ar}) ;{ }^{13} \mathrm{C}$ NMR $\left(75 \mathrm{MHz}, \mathrm{CDCl}_{3}\right) \delta_{\mathrm{C}}$ 9.2, 30.0, 44.8, 61.7, 70.8, 118.9,128.0, 128.2, 128.5, 128.8, 129.3, 135.1, 138.1, 142.0, 148.8, 165.4; LC-ESI-MS rt 10.9 min., m/z 353 (M+1), 375 (M+Na); IR (film): 3421, 2962, 2925, 2854, 1739, 1638, 1522, 1496, 1348, $1126 \mathrm{~cm}^{-1}$; Calcd for $\mathrm{C}_{20} \mathrm{H}_{20} \mathrm{~N}_{2} \mathrm{O}_{4}: \mathrm{C}, 68.17 ; \mathrm{H}, 5.72 ; \mathrm{N}$, 7.95. Found: C, 68.17; H, 5.69; N, 7.92.

\section{Oxidation of alcohol 5 to carbonyl derivative 6 or 7}

Compound 5 ( $1 \mathrm{mmol})$ and resin supported chromic acid $(3 \mathrm{mmol}, 3$ equiv., $1.5 \mathrm{~g}$. of resin with loading $2 \mathrm{mmol} / \mathrm{g})$ were refluxed in benzene $(10 \mathrm{~mL})$ for $24 \mathrm{~h}$. The resin was then filtered and solvent removed under reduced pressure. The carbonyl derivative was purified by flash chromatography on silica gel (cyclohexane/ethyl acetate $8 / 2$ as eluant).

(Z)-2-(1-Benzyl-2-oxo-4-phenylazetidin-3-ylidene)acetaldehyde $\left.\quad \mathbf{( 6}^{\mathbf{a}}\right) .{ }^{1} \mathrm{H} \quad \mathrm{NMR} \quad(300 \mathrm{MHz}$, $\left.\mathrm{CDCl}_{3}\right) \delta_{\mathrm{H}} 3.99\left(1 \mathrm{H}, \mathrm{d}, J=15.0 \mathrm{~Hz}, \mathrm{CH}_{2} \mathrm{Ph}\right), 4.98(1 \mathrm{H}, \mathrm{bs}, C H \mathrm{Ph}), 5.00(1 \mathrm{H}, \mathrm{d}, J=15.0 \mathrm{~Hz}$, $\left.\mathrm{CH}_{2} \mathrm{Ph}\right), 5.88\left(1 \mathrm{H}, \mathrm{dd}, J=7.8 \mathrm{~Hz}, J_{1,3}=0.9 \mathrm{~Hz}, \mathrm{CH}=\mathrm{C}\right), 7.05-7.50(10 \mathrm{H}, \mathrm{m}, \mathrm{Ar}), 10.42(1 \mathrm{H}, \mathrm{d}$, $J=7.8 \mathrm{~Hz}, \mathrm{CH}=\mathrm{O}) ;{ }^{13} \mathrm{C} \mathrm{NMR}\left(75 \mathrm{MHz}, \mathrm{CDCl}_{3}\right) \delta_{\mathrm{C}} 44.8,61.4,126.1,126.4,127.4,127.8,128.2$, 128.6, 129.0, 134.2, 134.5, 140.8, 161.5, 189.5; LC-ESI-MS rt $11.3 \mathrm{~min} ., \mathrm{m} / \mathrm{z} 278(\mathrm{M}+1), 300$ $(\mathrm{M}+\mathrm{Na})$; Calcd for $\mathrm{C}_{18} \mathrm{H}_{15} \mathrm{NO}_{2}$ : C, 77.96; H, 5.45; N, 5.05. Found: C, 77.95; H, 5.41; N, 5.09.

(E)-2-(1-Benzyl-2-oxo-4-phenylazetidin-3-ylidene)acetaldehyde $\left.\quad \mathbf{( 6}^{\mathbf{a}}\right) .{ }^{1} \mathrm{H} \quad \mathrm{NMR} \quad(300 \mathrm{MHz}$, $\left.\mathrm{CDCl}_{3}\right) \delta_{\mathrm{H}} 3.93\left(1 \mathrm{H}, \mathrm{d}, J=15.0 \mathrm{~Hz}, \mathrm{CH}_{2} \mathrm{Ph}\right), 4.98\left(1 \mathrm{H}, \mathrm{d}, J=15.0 \mathrm{~Hz}, C_{2} \mathrm{Ph}\right), 5.23(1 \mathrm{H}, \mathrm{d}$, $\left.J_{1,3}=1.5 \mathrm{~Hz}, C H \mathrm{Ph}\right), 6.42\left(1 \mathrm{H}, \mathrm{dd}, J=7.3 \mathrm{~Hz}, J_{1,3}=1.5 \mathrm{~Hz}, C H=\mathrm{C}\right), 7.05-7.20$ (2H, m, Ar), 7.20$7.40(6 \mathrm{H}, \mathrm{m}, \mathrm{Ar}), 7.40-7.50(2 \mathrm{H}, \mathrm{m}, \mathrm{Ar}), 9.47(1 \mathrm{H}, \mathrm{d}, J=7.3 \mathrm{~Hz}, \mathrm{CH}=\mathrm{O}) ;{ }^{13} \mathrm{C} \mathrm{NMR}(75 \mathrm{MHz}$, $\left.\mathrm{CDCl}_{3}\right) \delta_{\mathrm{C}} 44.8,62.3,120.1,127.5,128.8,128.6,129.0,129.4,129.6,134.1,134.9,141.8$, 160.9, 189.1; LC-ESI-MS rt 10.9 min., m/z $278(\mathrm{M}+1), 300(\mathrm{M}+\mathrm{Na})$; Calcd for $\mathrm{C}_{18} \mathrm{H}_{15} \mathrm{NO}_{2}$ : C, 77.96; H, 5.45; N, 5.05. Found: C, 77.92; H, 5.46; N, 5.02.

(E)-1-Benzyl-3-(2-oxobutylidene)-4-phenylazetidin-2-one (7b). ${ }^{1} \mathrm{H}$ NMR (300MHz, $\left.\mathrm{CDCl}_{3}\right)$ $\delta_{\mathrm{H}} 0.90\left(3 \mathrm{H}, \mathrm{t}, J=7.2 \mathrm{~Hz}, \mathrm{CH}_{3} \mathrm{CH}_{2}\right), 2.43\left(2 \mathrm{H}, \mathrm{m}, \mathrm{CH}_{3} \mathrm{CH}_{2}\right), 3.85\left(1 \mathrm{H}, \mathrm{d}, J=15.0 \mathrm{~Hz}, \mathrm{CH}_{2} \mathrm{Ph}\right)$, $4.86\left(1 \mathrm{H}, \mathrm{d}, \mathrm{J}=15.0 \mathrm{~Hz}, \mathrm{CH}_{2} \mathrm{Ph}\right), 5.17(1 \mathrm{H}, \mathrm{s}, \mathrm{CHPh}), 6.60(1 \mathrm{H}, \mathrm{s}, \mathrm{CH}=\mathrm{C}), 7.10-7.50(10 \mathrm{H}, \mathrm{m}$, $\mathrm{Ar}) ;{ }^{13} \mathrm{C} \mathrm{NMR}\left(75 \mathrm{MHz}, \mathrm{CDCl}_{3}\right) \delta_{\mathrm{C}} 8.6,31.7,45.2,60.8,127.1,128.0,128.3,128.5,128.7$, 
128.9, 129.4, 135.1, 138.3, 143.8, 164.9, 198.2; Calcd for $\mathrm{C}_{20} \mathrm{H}_{19} \mathrm{NO}_{2}$ : C, 78.66; H, 6.27; N, 4.59. Found: C, 78.63; H, 6.27; N, 4.60.

\section{Acknowledgements}

We thank MIUR (PRIN 2004 and FIRB 2001), CNR-ISOF and University of Bologna (Funds for selected topics) for financial support.

\section{References and Footnotes}

1. For a review see: (a) Magid, R. M. Tetrahedron 1980, 36, 1901. (b) Toromanoff, E. Tetrahedron 1980, 36, 2809. (c) Paquette, L. A.; Stirling, C. J. M. Tetrahedron 1992, 48, 7383. For recent reviews on metal catalyzed allylic substitution reactions see: (d) Pfaltz, A.; Lautens, M. In Comprehensive Asymmetric Catalysis II; Jacobsen, E. N. ; Pfaltz, A.; Yamamoto, H. Eds; Springer-Verlag Telos, 1999; Ch. 24, 833. (e) Trost, B. M.; Crawley, M. L. Chem. Rev. 2003, 103, 2921.

2. (a) Cardillo,G.; Fabbroni, S.; Gentilucci, L.; Perciaccante, R.; Piccinelli, F.; Tolomelli, A. Synthesis 2005, 61. For the synthesis of alkenyl derivatives see: (b) Bose, A.K.; Spiegelman, G.; Manhas, M.S. Tetrahedron Lett. 1971, 12, 3167. (c) Torii, S.; Okumoto, H.; Sadakane, M.; Abdul Hai, A.K.M.; Tanaka, H. Tetrahedron Lett. 1993, 34, 6553. (d) Bose, A.K.; Manhas, M.S.; Ghosh, M.; Shah, M.; Raju, V.S.; Bari, S.S.; Newaz, S.N.; Banik, B.K.; Chaudhary, A.G.; Barakat, K.J. J. Org. Chem. 1991, 56 , 6968. (e) Manhas, M.S.; Ghosh, M.; Bose, A.K. J. Org. Chem. 1990, 55, 575. (f) Banik, B.K.; Manhas, M.S.; Newaz, S.N.; Bose. A.K. Bioorg. Med. Chem. Lett. 1993, 3, 2363. (g) Banik, B.K.; Manhas, M.S.; Robb, E.W.; Bose, A.K. Heterocycles 1997, 44, 405. (h) Manhas, M.S.; Banik, B.K.; Mathur, A.; Vincent, J. E.; Bose A.K. Tetrahedron 2000, 56, 5587.

3. Cardillo,G.; Fabbroni, S.; Gentilucci, L.; Perciaccante, R.; Piccinelli, F.; Tolomelli, A. Org. Lett. 2005, 4, 533.

4. Cardillo,G.; Fabbroni, S.; Gentilucci, L.; Perciaccante, R.; Tolomelli, A. Adv. Synth. Catal. submitted.

5. (a) Abell, A. D.; Oldham, D. Bioorg. Med. Chem. Lett. 1999, 9, 497. (b) Annunziata, R.; Benaglia, M.; Cinquini, M.; Cozzi, F.; Puglisi, A. Bioorg. Med. Chem. 2002, 10, 1813. (c) Haley, T. M.; Angier, S. J.; Borthwick, A. D.; Singh, R.; Micetich, R. G. Drugs 2000, 3, 512. (d) Mascaretti, O. A.; Boschetti, C. E.; Danelon, G. O.; Mata, E. G.; Roveri, A. A. Curr. Med. Chem. 1995, 1, 441 (e) Edwards, P. D.; Bernstein, P. R. Med. Res. Rev. 1994, 14, 127. (f) Laborde, G.; Pezzenati, P.; Yovaldi, O. A.; Mascaretti, R. C.; Rossi, J. P. Bioorg. Med. Chem. 2001, 9, 2113. 
6. (a) Okovytyya, S.; Gorb, L.; Leszczynsk, J. Tetrahedron 2002, 58, 8751. (b) Okovytyya, S.; Gorb, L.; Leszczynsk, J. Tetrahedron Lett. 2002, 43, 4215. (c) Bach, R. D.; Glukhovtsev, M. N.; Gonzalez, C. J. Am. Chem. Soc. 1998, 120, 9902. (d) Hanzlik, R. P.; Shearer, G. O. J. Am. Chem. Soc. 1975, 97, 5231.

7. The bromine rearrangement has been studied by changing several reaction conditions. When catalytic amount of benzoylperoxide was used to induce the rearrangement or in the presence of radical inhibitors, no reaction occurred. The radical mechanism is currently under investigation and will be reported in due course.

8. (a) Anklam, S.; Liebscher, J. Tetrahedron 1998, 54, 6369. (b) Otto, H. H.; Bergmann, H. J.; Mayrhofer, R. Arch. Pharm. 1986, 319, 203.

9. (a) McKittrick, B. A.; Ma, K.; Dugar, S.; Clader, J. W.; Davis, H. J.; Czarniecki, M.; McPhail, A. T. Bioorg. Med. Chem. Lett. 1996, 6, 1947. (b) Burnett, D. A.; Caplen, M. A.; Davis, H. J.; Burrier, R. E.; Clader, J. W. J. Med. Chem. 1994, 37, 1733. (c) Rosenblum, S. B.; Huynh, T.; Davis, H. R. Jr Tetrahedron 2000, 56, 5735. (d) Vaccaro, W. D.; Davis, H. R. Jr. Bioorg. Med. Chem. Lett. 1998, 8, 313.

10. The mixture of $\mathrm{E}$ and $\mathrm{Z}$ diastereomers have been separated by flash chromatography and the subtitution reactions were performed on pure or highly enriched diastereomer. The reactions of E-2c, E-2d were performed only on the pure major isomer.

11. For an estensive study on polymer supported reagents see: (a) Cainelli, G.; Contento, M.; Manescalchi, F.; Plessi, L.; Panunzio, M. Gazz. Chim. It. 1983, 113, 523. (b) Cainelli, G.; Contento, M.; Manescalchi, F.; Plessi, L. Gazz. Chim. It. 1982, 112, 461. (c) Cainelli, G.; Contento, M.; Manescalchi, F.; Plessi, L. J. Chem. Soc. Chem. Comm. 1982, 725 . (d) Cainelli, G.; Contento, M.; Manescalchi, F.; Mussatto, M. C. Synthesis 1981, 302 (e) Cainelli, G.; Manescalchi, F.; Panunzio, M. Synthesis 1979, 141-4 (f) Cainelli, G.; Manescalchi, F. Synthesis 1976, 472.

12. Cainelli, G.; Cardillo, G.; Orena, M.; Sandri, S. J. Am. Chem. Soc. 1976, 98, 6737. 